\title{
Voice Call Quality Using 802.11E On A Wireless Mesh Network
}

\author{
by \\ David Alexander van Geyn
}

\author{
A thesis submitted to the \\ School of Computing \\ in conformity with the requirements for \\ the degree of Master of Science
}

Queen's University

Kingston, Ontario, Canada

May 2008

Copyright (c) David Alexander van Geyn, 2008 


\section{Abstract}

Wireless Local Area Networks (WLANs) provide an affordable solution for last mile network access. They also allow for extension of a network by configuring a Wireless Mesh Network (WMN) where it may otherwise be physically infeasible or cost prohibitive to do so. With the increasing use of real-time applications such as video conferencing and Voice over IP (VoIP), networks are stressed to guarantee Quality of Service (QoS) requirements for these applications. Examples of key requirements include bounded delay and packet loss ratios. Addressing this issue in Wireless Local Area Networks (WLANs), the IEEE 802.11e amendment was proposed to provide a QoS mechanism. However, the performance of 802.11e in meshed environments is yet to be studied.

In this work, we study Voice over Internet Protocol (VoIP) call quality in a meshed environment with provisions for QoS. We study the call quality and throughput of background traffic in an experimental WMN testbed in order to test how well the IEEE 802.11e QoS provisions support voice calls. Call quality is tested in different configurations and scenarios. We study the effect of the number of wireless hops on VoIP call quality. In addition, we investigate the number of VoIP calls that can be supported simultaneously for different numbers of wireless hops. We also study how fairly the network treats different calls in different configurations. Then, we look at 
how much effective bandwidth a VoIP call uses on the network. Finally, we examine the VoIP call quality of different calls when calls have different QoS parameters and study the effect that a busy central node has on traffic passing through it. We provide suggestions to improve call quality on a WMN and hint at possible future work. 


\section{Acknowledgments}

I would like to thank Queen's University at Kingston and the School of Computing for giving me the opportunity and financial assistance to pursue the degree of Master of Science. I am also thankful to my supervisor, Dr. Hossam Hassanein for allowing me to be a part of the Telecommunications Research Lab, Mobile Services Group, and to work under his guidance. Dr. Hassanein provided invaluable assistance, direction and motivation that helped to complete the work.

I am also thankful to Dr. Abd-Elhamid Taha for his invaluable assistance in reviewing the work. His comments and feedback were extremely helpful and greatly assisted in producing a high quality work.

It has been an enjoyable time working in the Telecommunications Research Lab, and I will miss it. My thanks go out to everyone in the School of Computing and the lab for their support. 


\section{Table of Contents}

Abstract $\quad$ i

Acknowledgments

Table of Contents $\quad$ iv

List of Tables $\quad$ vi

List of Figures vii

List of Acronyms $\quad$ ix

Chapter 1:

Introduction ................. 1

1.1 Contribution of Thesis . . . . . . . . . . . . . . . . . . . 3

1.2 Organization of Thesis . . . . . . . . . . . . . . . 4

Chapter 2:

Background ................ 5

2.1 IEEE $802.11 \ldots \ldots \ldots \ldots \ldots$

2.2 Voice over Internet Protocol . . . . . . . . . . . . . . . . . . . 12

2.3 Signaling Protocols . . . . . . . . . . . . . . . . . . . . . . . . . . . . . . . . . . . 15

2.4 Wireless Mesh Networks . . . . . . . . . . . . . . . . . 17

2.5 Related Performance Works . . . . . . . . . . . . . . . . . . . 19

2.6 Summary . . . . . . . . . . . . . . . . . . 22

Chapter 3:

Implementation Details . . . . . . . . . . . 24

3.1 Experimental Setup . . . . . . . . . . . . . . . . . . . . . . . . . . . . . . . 24

3.2 Measuring Call Performance . . . . . . . . . . . . . . . . . . 32

3.3 Gathering, Processing, and Use of Experimental Data . . . . . . . . . 34

3.4 Summary . . . . . . . . . . . . . . . . . 38 
Chapter 4:

Performance Evaluation . . . . . . . . . . . . 39

4.1 Experimental Results . . . . . . . . . . . . . . . . . . . 39

4.2 Summary . . . . . . . . . . . . . . . . . . . 74

Chapter 5:

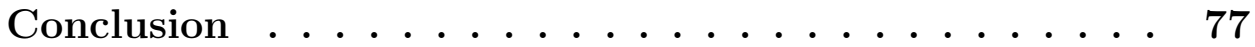

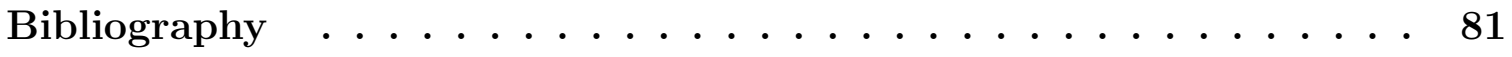

Appendix A:

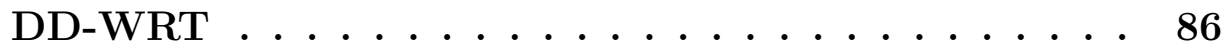

Appendix B:

E-model .................. 89 


\section{List of Tables}

2.1 Default EDCA Parameters . . . . . . . . . . . . . . . . . . . 11

2.2 VoIP Codec Options . . . . . . . . . . . . . . . . . . 13

3.1 DD-WRT Mesh Router Settings . . . . . . . . . . . . . . 29

4.1 Average Call Qualities with Different CWmax Settings . . . . . . . . 47

4.2 Average Call Quality Comparison of $63 \mathrm{~ms}$ CWmax with and without AIFS Adjustment . . . . . . . . . . . . . . . . . 50

4.3 Bandwidth Available for Background Traffic With Calls Added to the Network . . . . . . . . . . . . . . . . 53

4.4 Results of Fairness Test of Calls on Separate Portions of the Network 56

4.5 Results of Fairness Test of Calls on Separate but Touching Portions of the Network . . . . . . . . . . . . . . . . . . 57

4.6 Results of Fairness Test of Calls on Separate but Touching Portions of the Network . . . . . . . . . . . . . . . . . . 58

4.7 Results of Fairness Test of Calls with One Hop Overlap . . . . . . . . 59

4.8 Results of Fairness of Maximum Supported Calls Test . . . . . . . . . 60

4.9 Results of Fairness Test of Calls with Calls of Different Hop Counts . 61

4.10 Results of Available Bandwidth Test with Two Hops Separation . . . 63

4.11 Results of Available Bandwidth Test with One Hop Separation . . . . 64

4.12 Results of Available Bandwidth Test with No Separation (Adjacent) . 65

4.13 Results of Available Bandwidth Test with No Separation (Adjacent) but Four Hops . . . . . . . . . . . . . . . . . . . 66

4.14 Results of Call Test, Overlapping Calls, Different QoS Settings . . . . 68

4.15 Results of Call Test, Separate Calls, Different QoS Settings . . . . . . 70

4.16 Results of Call Test, Adjacent Calls, Different QoS Settings . . . . . . 71

4.17 Results of Call Test, Bottleneck Node . . . . . . . . . . . . . . . . . 73

4.18 Maximum Number of Supported Calls . . . . . . . . . . . . . . . . 74 


\section{List of Figures}

2.1 Typical Wireless Local Area Network . . . . . . . . . . . . . 6

2.2 Two stations unable to hear one another . . . . . . . . . 8

2.3 Typical Wireless Mesh Network Configuration . . . . . . . . . 18

3.1 General Mesh Network Testbed Configuration . . . . . . . . . . . 29

3.2 Router Placement . . . . . . . . . . . . . . . . . . . . . . . . 30

3.3 Diagram of an Asterisk setup _ . . . . . . . . . . . . . . . 32

3.4 X-Lite Interface . . . . . . . . . . . . . . . . . . . . . . . . . . 35

3.5 Wireshark Interface . . . . . . . . . . . . . . . . . 36

4.1 Diagram of 5 Hop Configuration . . . . . . . . . . . . . . . 41

4.2 MOS of 1 and 2 calls, with and without background traffic, no traffic has priority . . . . . . . . . . . . . . . . 42

4.3 MOS of 1 and 2 calls, with and without background traffic, CWmax $7 \quad 43$

4.4 MOS of 1 and 2 calls, with and without background traffic, CWmax 1544

4.5 MOS of 1 and 2 calls, with and without background traffic, CWmax $63 \quad 45$

4.6 MOS of 1 and 2 calls, with and without background traffic, CWmax

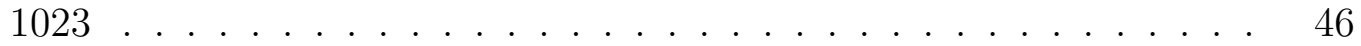

4.7 Throughput of Background Traffic with Varying CWmin Settings . . 47

4.8 Call Quality With Increased Background Traffic AIFS Values . . . . . 48

4.9 MOS of 1 and 2 calls, with and without background traffic, CWmax 63 , AIFS $15 \ldots \ldots \ldots \ldots \ldots$

4.10 Diagram of 5 Hop Configuration for Maximum Supported Calls Tests 51

4.11 Average MOS of Different Numbers of Calls at Different Hop Counts 52

4.12 Diagram for Fairness of Two Calls on Separate Portions of the Network 55

4.13 Diagram for fairness of two calls on separate but touching portions of the network . . . . . . . . . . . . . . 56

4.14 Diagram for fairness of three calls, with two overlapping calls and one separate . . . . . . . . . . . . . . . 57

4.15 Diagram for fairness of two calls, with two overlapping calls . . . 58

4.16 Diagram for fairness of two calls, with two overlapping calls of different hop counts . . . . . . . . . . . . . . . . . 61 
4.17 Diagram showing background traffic and call, separated by two hops . 62

4.18 Diagram showing background traffic and call, separated by a single hop 63

4.19 Diagram showing background traffic and call, separated by no space . 64

4.20 Diagram showing background traffic and call, separated by no space . 65

4.21 Diagram showing three overlapping calls . . . . . . . . . . . 67

4.22 Diagram showing two calls running on separate parts of the network . 69

4.23 Diagram showing two calls that are adjacent to one another . . . . 70

4.24 Diagram showing three calls that all pass through a central node . . . 72

A.1 DD-WRT Wireless Configuration Page . . . . . . . . . . . . 87

A.2 DD-WRT QoS Configuration . . . . . . . . . . . 88

B.1 MOS as a Function of the R-Value . . . . . . . . . . . . . . . . . 92 


\section{List of Acronyms}

\begin{tabular}{|c|c|}
\hline ACK & Acknowledgment \\
\hline AIFS & Arbitration Interframe Space \\
\hline CFP & Contention Free Period \\
\hline CODEC & Compressor/Decompressor \\
\hline $\mathrm{CP}$ & Contention Period \\
\hline CSMA/CA & Carrier Sense Multiple Access with Collision Avoidance \\
\hline CSV & Comma Separated Value \\
\hline CTS & Clear To Send \\
\hline CWmax & Maximum Contention Window Size \\
\hline CWmin & Minimum Contention Window Size \\
\hline DCF & Distributed Coordination Function \\
\hline DiffServ & Differentiated Services \\
\hline DSCP & Distributed Services Code Point \\
\hline EDCA & Enhanced Distributed Channel Access \\
\hline FTP & File Transfer Protocol \\
\hline HCCA & Hybrid Coordinator Function Controlled Channel Access \\
\hline HCF & Hybrid Coordinator Function \\
\hline HTTP & Hypertext Transfer Protocol \\
\hline IP & Internet Protocol \\
\hline IVR & Interactive Voice Response \\
\hline MAC & Medium Access Control \\
\hline
\end{tabular}




$\begin{array}{ll}\text { MGCP } & \text { Media Gateway Control Protocol } \\ \text { MOS } & \text { Mean Opinion Score } \\ \text { NAV } & \text { Network Allocation Vector } \\ \text { PBX } & \text { Private Branch Exchange } \\ \text { PC } & \text { Point Coordinator } \\ \text { PCF } & \text { Point Coordination Function } \\ \text { PHY } & \text { Physical } \\ \text { QoS } & \text { Quality of Service } \\ \text { SIP } & \text { Session Initiation Protocol } \\ \text { RFC } & \text { Request For Comments } \\ \text { RTCP } & \text { Real Time Control Protocol } \\ \text { RTP } & \text { Real Time Protocol } \\ \text { RTS } & \text { Request To Send } \\ \text { TCP } & \text { Transmission Control Protocol } \\ \text { TXOP } & \text { Transmit Opportunity } \\ \text { VoIP } & \text { Voice over Internet Protocol } \\ \text { UDP } & \text { User Datagram Protocol } \\ \text { WDS } & \text { Wireless Distribution System } \\ \text { WLAN } & \text { Wireless Local Area Network } \\ \text { WiMA } & \text { Worldwide Interoperability for Microwave Access } \\ \text { WA } & \text { Wetwork } \\ \text { WA Network }\end{array}$




\section{Chapter 1}

\section{Introduction}

Wireless networks have revolutionized the telecom industry and have allowed for customer mobility on many different networks. Cellular, Wireless Local Area Networks (WLANs), and Worldwide Interoperability for Microwave Access (WiMAX) are just a few types of wireless networks in use today. With the convergence of different communication methods to Internet Protocol (IP) based networks, it is understood that different kinds of media have different requirements. That is, services such as E-mail, file transfer, and web browsing have different delay tolerances than, say, Voice over Internet Protocol (VoIP). While transfer speed is important to E-mail and file transfer services, both can tolerate delays if needed. Web browsing is becoming more interactive and thus cannot tolerate as much delay as E-mail or file transfer services, but can still tolerate some delay without difficulty. Video conferencing is an application with requirements that fit somewhere in between VoIP and file transfer. That is, it can adapt to variable network conditions by adjusting the video quality upwards or downwards while keeping the voice quality constant. While this is not ideal, it reduces the amount of bandwidth a video conference uses. When comparing VoIP 
and file transfers, VoIP cannot tolerate large amounts of delay or loss, and as such, is one of the most demanding services on an IP network.

Needless to say, VoIP is a useful service and is becoming widespread. It is projected that the percentage of VoIP lines being installed in the corporate sector will reach $44 \%$ by the end of 2008 [12]. Motivations for using VoIP include the cost savings and ease of deployment since the existing data network infrastructure can be used. Alternatives to VoIP in an office environment are costly proprietary Private Branch Exchange (PBX) systems with exclusive wiring for each phone.

Due to the growing use of both WLANs and VoIP, VoIP over wireless networks is receiving wide interest. Using wireless VoIP can provide a user with limited mobility and the convenience of using a portable phone, while not being tied to a computer or desk phone. Some cellular telephones also have the ability to connect to WLANs, therefore providing a user with two network types in one device. This allows for VoIP use when in range of a WLAN, and cellular network use otherwise.

Providing wireless VoIP users with phone services over a wide area requires good WLAN coverage. This coverage can be provided and extended by use of Wireless Mesh Networks (WMNs). WMNs, unlike traditional wireless networks where each access point is connected to the wired network, allow all of the access points to be connected to the wired network through each other. A WMN is similar to the wired network in that routers forward packets from one node to the next. In a WMN, however, the packets are forwarded from access point to access point through the wireless medium until the destination is reached. The destination can be on another network, in which case the data travels over the mesh network to the wired part and beyond. It can also be on the WMN, in which case the data travels only over the 
WMN. Regardless, data must traverse the mesh network and the network must be able to handle a variety of services successfully.

Providing real-time services such as voice over a wireless network is difficult given that no QoS mechanism was provided in the original IEEE 802.11 [32] standard. The 802.11e amendment to the IEEE 802.11 [32] standard adds a QoS mechanism to 802.11 WLANs. 802.11e has been previously evaluated in a non-meshed environment, but has not been evaluated on a WMN testbed. We discuss 802.11e in Chapter 2 . In this thesis, we examine how well 802.11e supports voice calls on an experimental WMN testbed.

\subsection{Contribution of Thesis}

In this thesis, we focus on the functionality of 802.11e in providing desired QoS for voice calls on a WMN. We will evaluate call quality in a variety of configurations and scenarios on a WMN testbed. The thesis provides these main contributions:

- Identifying performance metrics for VoIP calls that will allows the comparison of call-to-call quality.

- Creating novel test scenarios to evaluate VoIP over a WMN, as well as scenarios that test different characteristics, such as: effect on background traffic, fairness among calls, maximum number of calls supported, etc.

- Establish guidelines for configuring parameters of 802.11 WMNs to help increase call quality.

In order to measure voice quality, we will be recording data to calculate end-to-end delay, jitter and packet loss. We will use this data to calculate a Mean Opinion Score 
(MOS) value that represents perceived voice quality. This will be calculated through the use of the ITU-T E-model [37], a method of objectively calculating perceived voice quality for use in network planning and evaluation. The E-model calculates perceived voice call quality and provides a way to calculate a MOS.

\section{$1.2 \quad$ Organization of Thesis}

The rest of the thesis is organized as follows. In Chapter 2, we discuss the IEEE 802.11 wireless standard and provide background information on WMNs, VoIP, performance metrics of VoIP, and Session Initiation Protocol (SIP) - the signaling protocol used for our VoIP calls. We also discuss some other voice call performance studies that deal with voice call quality on wireless networks. In Chapter 3, we provide implementation details of our WMN testbed. This includes discussion on the software used, the mesh network configuration, call quality information, as well as the methods used for collecting and processing the experimental data. We also discuss how the testing is performed. In Chapter 4, we detail the specifics of the different test scenarios and present and summarize the results. In Chapter 5, we suggest ways of improving the performance of WMNs with respect to voice call quality. We also suggest future research directions in addressing voice call quality over WMNs. 


\section{Chapter 2}

\section{Background}

In this chapter, we provide an overview of the IEEE 802.11 standard. We then discuss the IEEE 802.11e QoS mechanisms. Specifically, how they can improve performance for different classes of Internet traffic. The traffic classes defined are background, best effort, video and voice. Following this, we discuss the use of IEEE 802.11 as WLANs. We then discuss VoIP and the performance metrics used to calculate voice call quality, as well as SIP, the signaling protocol used for our voice calls. We then discuss WMNs and their applications. We will also briefly discuss other performance studies that relate to our work on voice call quality over wireless.

\section{$2.1 \quad$ IEEE 802.11}

The IEEE 802.11 standard [32], like with other 802.x protocols, defines the Medium Access Control (MAC) and Physical (PHY) layers for 802.11. We are concerned with the MAC layer specifications, as these specifications apply to our research. Defined are two different methods of accessing the wireless medium, the Distributed Coordination 
Function (DCF), and the Point Coordination Function (PCF). The DCF is implemented using Carrier Sense Multiple Access with Collision Avoidance (CSMA/CA). The PCF access method uses a Point Coordinator (PC) function to determine which station can transmit at any given time. The PCF is not widely implemented, as is discussed later in this chapter.

A typical 802.11 wireless network consists of access points and wireless stations. An access point is a device that wireless stations connect to in order to gain access to a network. Access points are typically stationary while wireless stations can be mobile. Figure 2.1 shows a schematic of a typical 802.11 wireless network.

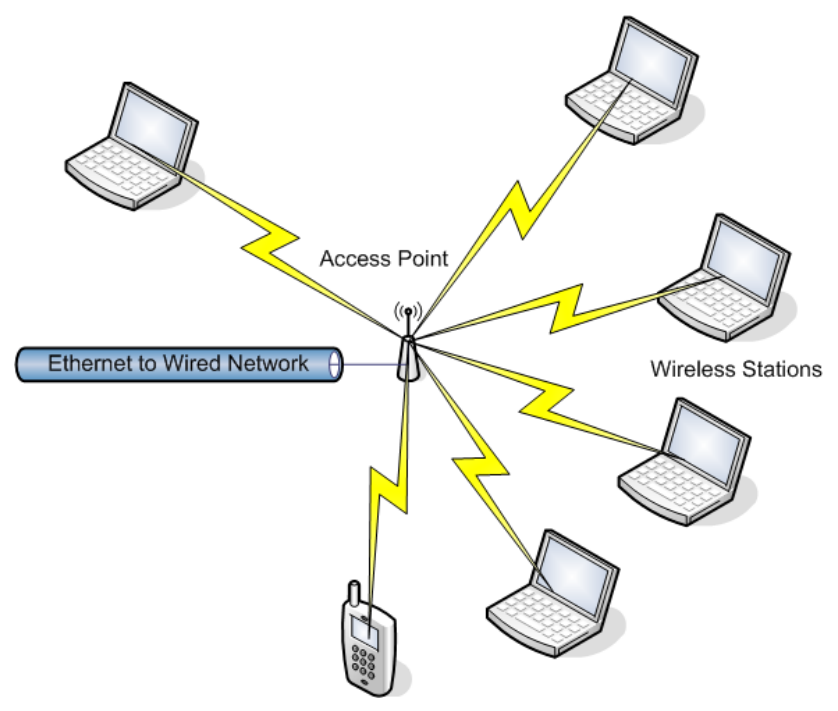

Figure 2.1: A typical wireless local area network configuration.

An 802.11 amendment, 802.11a, uses the unlicensed $5 \mathrm{GHz}$ radio band and can operate at as high a rate as $54 \mathrm{Mbps}$. The $802.11 \mathrm{~b}$ amendment provides a rate of 11 Mbps using the unlicensed $2.4 \mathrm{GHz}$ radio band. With $802.11 \mathrm{~g}$, the maximum rate was increased to $54 \mathrm{Mbps}$ while still using the $2.4 \mathrm{GHz}$ radio band. The $802.11 \mathrm{~b}$ 
and 802.11g amendments are compatible with one another, and most wireless 802.11 devices today support both. Support for 802.11a, on the other hand, is not as widely available.

\subsubsection{Distributed Coordination Function}

A wireless station that uses the DCF method of medium access uses CSMA/CA as the carrier sensing protocol. Using this method, the station senses when the medium is free from other stations transmitting, and decides when it should attempt to transmit. The CSMA part of the protocol works as follows. When a station is ready to transmit, it listens to the wireless medium. If the medium is free, determined by sensing for other transmitting stations, then the station will transmit. If the medium is not free, the station will wait a period of time before trying again. With this protocol, there is a chance that multiple stations that have been waiting to transmit may simultaneously attempt to transmit when the medium becomes free. A random backoff timer helps to alleviate this simultaneous transmission problem (also called a collision) by having stations wait different amounts of time before transmitting. When a collision is detected, a random backoff timer helps to prevent a collision at the next transmission attempt. This method works well when all stations can hear one another; but, with the wireless medium, this is not always the case. Two stations on opposite ends of the transmission/hearing range of an access point will both be able to hear the access point, but may not be able to hear each other, in which case they may transmit at the same time and cause a collision. Accordingly, to reduce the chance of collisions, the DCF exercises a virtual carrier sense mechanism. 


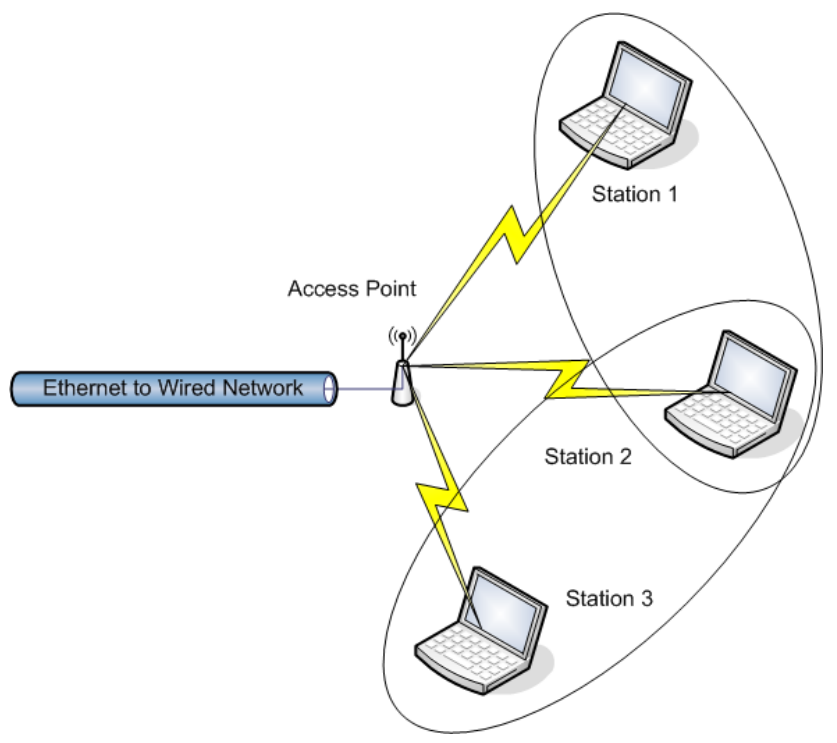

Figure 2.2: A diagram showing two stations that are unable to hear one another (Station 1 and Station 3). RTS/CTS solves this problem.

The virtual carrier sense mechanism requires stations wanting to transmit to send a Request To Send (RTS) control packet. This packet contains the source station identifier, destination station identifier and expected transmission duration of the packet and associated Acknowledgment (ACK) packet. If the medium is free, the destination sends a Clear To Send (CTS) packet. All stations within the hearing range then know that the wireless medium is in use for the length of time requested by the RTS packet. The virtual carrier sense indicator, called a Network Allocation Vector (NAV), is then set based on this information. Since other stations know how long a station is expected to transmit, they wait this amount of time until trying to transmit. The purpose of the RTS/CTS mechanism is to reduce the chance of collisions when there are many active stations on a 802.11 wireless network, and to help prevent the situation above, where two stations that cannot hear each other cause collisions. 


\subsubsection{Point Coordination Function}

The PCF is based on a polling scheme, which can be implemented using either a round-robin or more complex priority-based scheme. When using PCF, the wireless channel has a repetitious frame structure that consists of a Contention Free Period (CFP) and a Contention Period (CP).

During each CFP, an access point's PC steps through all stations currently operating in PCF mode. After each station is polled, an opportunity to transmit data is granted if the station wishes to transmit.

The 802.11 standard is vague in defining some parts of the PCF. As a result, a PCF implemented by one vendor may not be interoperable with a PCF implemented by another vendor. Accordingly, the PCF was not widely provided commercially and will thus not be considered in our further discussions.

\subsection{1e}

With the increasing use of video conferencing applications and VoIP applications, QoS support was needed for 802.11 wireless networks. The 802.11e specification, approved by the IEEE in 2005, defines QoS mechanisms for 802.11 wireless equipment. It is now part of the 2007 edition of the 802.11 standard [32]. The purpose of the specification is to better support bandwidth and delay sensitive applications such as VoIP and video conferencing, while maintaining backward compatibility with stations that do not support the enhancements provided by the 802.11 e specification.

The enhancement to the DCF, Enhanced Distributed Channel Access (EDCA), introduces traffic categories. In order, from highest priority to lowest priority, they are, voice traffic, video traffic, best effort traffic and background traffic. Each traffic 
category has five adjustable parameters.

1. Minimum Contention Window Size (CWmin)

2. Maximum Contention Window Size (CWmax)

3. Arbitration Interframe Space (AIFS)

4. Transmit Opportunity Length $(\mathrm{TXOP}(\mathrm{b}))$

5. Transmit Opportunity Length $(\mathrm{TXOP}(\mathrm{a} / \mathrm{g}))$

The CWmin value represents the upper limit in milliseconds of the random choice for the initial wait time before transmission. The number chosen will be between 0 and the CWmin value, inclusive. If the wait time expires before the medium is idle, the wait time is doubled. This process continues until either the packet is transmitted or the value of CWmax is reached. Once the CWmax value is reached, retries are attempted at the CWmax wait time until conditions improve.

The AIFS parameter, also in milliseconds, is the amount of time a packet will wait before beginning the process of calculating the random backoff time. It is a way of allowing higher priority traffic to have a better chance of being transmitted first by having a smaller AIFS parameter. The higher priority the queue, the smaller the AIFS parameter should be set.

The TXOP parameter allows for setting the interval of time that a station can transmit. The more delay-sensitive the traffic, the smaller the TXOP interval should be. This is because more delay-sensitive traffic transmits smaller packets more frequently, so the actual time needed to transmit a packet is smaller. A TXOP interval 
of zero, as in the case of background and best effort traffic, means that there is no limit imposed by EDCA. Table 2.1 shows the default EDCA parameters.

Table 2.1: Default EDCA Parameters

\begin{tabular}{|c|c|c|c|c|c|}
\hline & CWmin & CWmax & AIFSN & TXOP(b) & TXOP(a/g) \\
\hline Background & 15 & 1023 & 3 & 0 & 0 \\
\hline Best Effort & 15 & 63 & 3 & 0 & 0 \\
\hline Video & 7 & 15 & 1 & 6016 & 3008 \\
\hline Voice & 3 & 7 & 1 & 3264 & 1504 \\
\hline
\end{tabular}

The Hybrid Coordinator Function (HCF) Controlled Channel Access (HCCA) specification is an amendment to 802.11 that is similar to the PCF in that access points must coordinate access to the medium. This is done by supporting scheduling and queueing within the access point. Stations running in this mode must be able to respond to poll messages from the access point. Few access points currently support the HCCA mode of access. It is not used in our research since it is not implemented in the majority of 802.11 devices.

Packets that are to be given higher priority than other packets must be tagged at the network (IP) layer. The tagging mechanism used is from the Differentiated Services (DiffServ) model, defined in RFC 2474 [27] and 2475 [5]. The DiffServ architecture defines a field in the IP header to make per-hop decisions about how to handle packets with respect to prioritization. The six most significant bits of a one byte field in the IP header are used as the Distributed Services Code Point (DSCP). Two bits remain that are not used by the DSCP. The DSCP is set to Ox2f (101111) for voice calls. Normal background traffic is the default, $0 x 00$. This IP header field is looked at by routers in the network and the packet is handled and queued accordingly. 


\subsection{Voice over Internet Protocol}

VoIP usage has ballooned in recent years, both for residential and office users. Managing a single network for both voice and data is one incentive to deploying VoIP, as is the ability to have more information available on a telephone's display. This information can include a synchronzied contact list, e-mails, notes and tasks.

The VoIP protocol suite can be broken down into two categories: control protocols and data protocols. The control protocols used with VoIP are used to set up and tear down calls, maintain voice traffic, handle key presses on the telephones, and maintain network operation. These protocols include SIP and Real Time Control Protocol (RTCP). The data protocols used with VoIP are used to transport the actual voice data, that is, the digitized voice data.

VoIP uses both the Transmission Control Protocol (TCP) and User Datagram Protocol (UDP) protocols for transport. The control protocols use TCP, while the data protocols and voice traffic use UDP. TCP is used for the control protocols because it is a lossless protocol; lost packets are recovered and delivery is guaranteed. Voice traffic uses UDP because guaranteed delivery is not required. There is no reason to have lost packets delivered at a later time in a conversation.

The voice data is sent using Real Time Protocol (RTP) over UDP. Each RTP packet contains a short sample of the voice conversation, ranging from $10 \mathrm{~ms}$ up to $50 \mathrm{~ms}$. The size of the RTP packet and the length of the voice sample depends on the Compressor/Decompressor (CODEC) used for the voice conversation. In the WMN testbed, we use $20 \mathrm{~ms}$ sampling time, which is the most common sampling time used for VoIP use [37].

To send voice data over the network, it must be passed through a CODEC. Many 
CODECs are available for VoIP use. The most common CODECs and a brief description are listed in Table 2.2.

Table 2.2: VoIP Codec Options

\begin{tabular}{|c|l|}
\hline CODEC Name & Description \\
\hline G.711 & $\begin{array}{l}\text { Produces a } 64 \text { kbps stream, low CPU utilization. Considered } \\
\text { the highest quality. }\end{array}$ \\
\hline G.723.1 & Low bit rate of 5.3 or $6.4 \mathrm{kbps}$, reasonable quality. \\
\hline G.726 & Designed for packet to PBX interconnections. \\
\hline G.728 & Produces a 16 kbps stream, considered reasonable quality. \\
\hline G.729 & Produces an 8 kbps stream, considered good quality. \\
\hline
\end{tabular}

The G.711 CODEC is a widely used CODEC and is used on T1 PRI connections to digital PBX systems as well as for VoIP. With this type of system, a T1 PRI provides 23 channels of 64 kbps each. Each channel can handle one G.711 call. The other CODECs are used in some VoIP implementations. G.729 is one of the more popular ones for its low bandwidth use but good quality. The G.711 CODEC is, however, the best quality and best sounding CODEC in use for VoIP.

\subsubsection{Voice Quality Metrics}

Several factors can affect the quality of a voice call. One key factor is the choice of CODEC. Choosing a poor quality CODEC will result in a poor quality voice call, even if all other conditions are perfect for a high quality voice call. Generally speaking, a higher bit-rate CODEC will produce a higher quality representation of voice. The G.711 codec is uncompressed, and is considered the best possible CODEC choice for a voice call. The downside to G.711 is that it uses more bandwidth than all the others, 
at $64 \mathrm{kbps}$ per direction. On a local network, the higher bandwidth is not a problem. While CODEC choice does have an impact on call quality, this loss of quality due to CODEC choice is known for each CODEC and does not change.

The biggest changing factor in call quality is the design, configuration and utilization of the network the voice calls are traversing. A network can affect a voice call by introducing delay, jitter, and packet loss. Any or all of these impairments can contribute to voice call quality.

Delay is the total time it takes for the voice to be encoded and transmitted on one end, and received and decoded on the other end. It includes CODEC processing time at both ends, jitter buffer in the phone, and the time it takes for the voice packets to traverse the network from one end to the other. A large delay can make conversation seem like a two-way radio and make it difficult to communicate.

Jitter is caused by changes in the inter-arrival gap at the endpoint, that is, if voice packets are being encoded once every $20 \mathrm{~ms}$, the ideal inter-arrival gap at the endpoint is $20 \mathrm{~ms}$. The deviation from the ideal gap is the instantaneous jitter. That is, if all packets arrive exactly every $20 \mathrm{~ms}$, instantaneous jitter is always $0 \mathrm{~ms}$. If the inter-arrival gap varies every ten packets, then the instantaneous jitter will be $0 \mathrm{~ms}$ except for during the variation every ten seconds. This gives a result with a spike every ten seconds. To solve this problem, the RTP specification in RFC 3550 [31] describes the recommended algorithm for calculating jitter. A low-pass filter is applied to the instantaneous jitter in order to smooth the reported jitter value. This is done as described in the following formulae.

$$
\begin{gathered}
j_{1}=\text { first instantaneous jitter result } \\
j_{i}=\frac{15}{16} j_{i-1}+\frac{1}{16}\left|j_{i}\right|, \text { where } \mathrm{i}=2, \ldots \mathrm{N}
\end{gathered}
$$


To overcome the variation in jitter, a jitter buffer is implemented in phones to buffer incoming packets for a short period of time to allow for slight variations in the inter-arrival gap without audible problems.

Packet loss is the actual loss of packets through a network. Typically, packet loss is caused by overutilized links or poor quality links that experience numerous phyiscal layer errors. A high packet loss rate makes voice calls annoying to listen to.

\subsection{Signaling Protocols}

Control protocols are used for signaling in VoIP and allow user devices, such as software phones or IP phones to set up and tear down calls. There are numerous signaling protocols available, including SIP, H.323, and Media Gateway Control Protocol (MGCP). SIP is widely implemented in software phones and VoIP devices. H.323 is also widely used and was the first widely adopted signaling protocol for VoIP. MGCP is not in as widespread use, but is used at interconnection points between VoIP and traditional telephone networks. Due to its widespread use, we have selected SIP as the signaling protocol in this research. SIP is discussed in further detail in the following subsection. Ultimately, choice of signaling protocol does not affect call quality.

\subsubsection{Session Initiation Protocol}

SIP, defined in RFC 2543 [17] and 3261 [30], is intended to set up, tear down and manage multimedia sessions. It can be used with any type of multimedia session, such as data sharing, video conferencing, and VoIP. The majority of VoIP devices 
on the market all support SIP, including: hardware IP phones, software IP phones, analog terminal adapters, IP PBX systems, and others. It is the standard that many manufacturers are using to develop new products.

Hypertext Transfer Protocol (HTTP) is used for normal web browsing, and is based on a request-response model. The web browser connects to a web server, sends a request, and receives a response which is processed and displayed on screen. Advantageous features of SIP include a HTTP-similar model and the ability to handle multiple types of media sessions in one call. The first feature allows for user mobility without changing the user's address. The second feature allows users to engage in a voice call, a game, and instant messaging all at the same time within the same SIP call.

SIP distinguishes between two different device types, user agents and network devices. The user agents are the user devices, such as a hardware phone, software phone or video conferencing device. Network devices are the servers that handle redirection, proxy services and registration. Redirection servers re-route a SIP request to the appropriate location, proxy servers can act as brokers across a firewall, and registration servers handle new client registrations and registration updates.

As mentioned above, the SIP protocol is similar to HTTP because it is also based on a request-response model. With this type of model, a client sends a message to a server, and a server responds to a client. Each message has a header which contains the message type, caller, and callee. Requests that a client can send include invite, acknowledgement, bye, options, cancel and register messages. The purpose of each message is as follows:

- Invite - a request from a client to speak to another client. It contains client 
capabilities and media type, along with a header that contains the caller and callee.

- Acknowledgment - a possible response to an invite and represents a successful connection. It is after the acknowledgment message that the voice, video or data exchange begins.

- Bye - used to terminate a call.

- Options - used to collect and send information about clients and servers.

- Cancel - used to cancel a message exchange that is in progress but has not yet been completed.

- Register - used to register clients with servers. By registering, clients are then able to use the services that a particular server offers, whether voice call termination, video conferencing services, or others.

SIP servers respond to the request messages with either a provisional or final message. Provisional messages are messages that are sent during a request/response process in order to provide status updates. A final message is the last response to a series of request/response messages, and conveys the result of the processing.

\subsection{Wireless Mesh Networks}

WMNs are communication networks made up of two types of nodes: mesh routers and mesh clients. Mesh routers forward packets to one another and route data from source to destination using the wireless medium. In most cases, WMNs are used for 
last mile access, and as a result, the majority of data that travels over a WMN is sent from a client, across the network to a gateway node and the same in reverse. A gateway node is a mesh router that also has access to another network, wired or wireless, such as a local area network. Mesh routers are predominantly fixed in place, while mesh clients are mobile and can come and go without affecting the operation of the WMN. The advantages provided by WMNs include low installation cost and extension of a wireless network without a wired backhaul. Installation of a wired backhaul may be cost prohibitive or physically infeasible, so extension of a network by using a WMN saves on the up-front deployment cost. Figure 2.3 shows a typical WMN configuration.

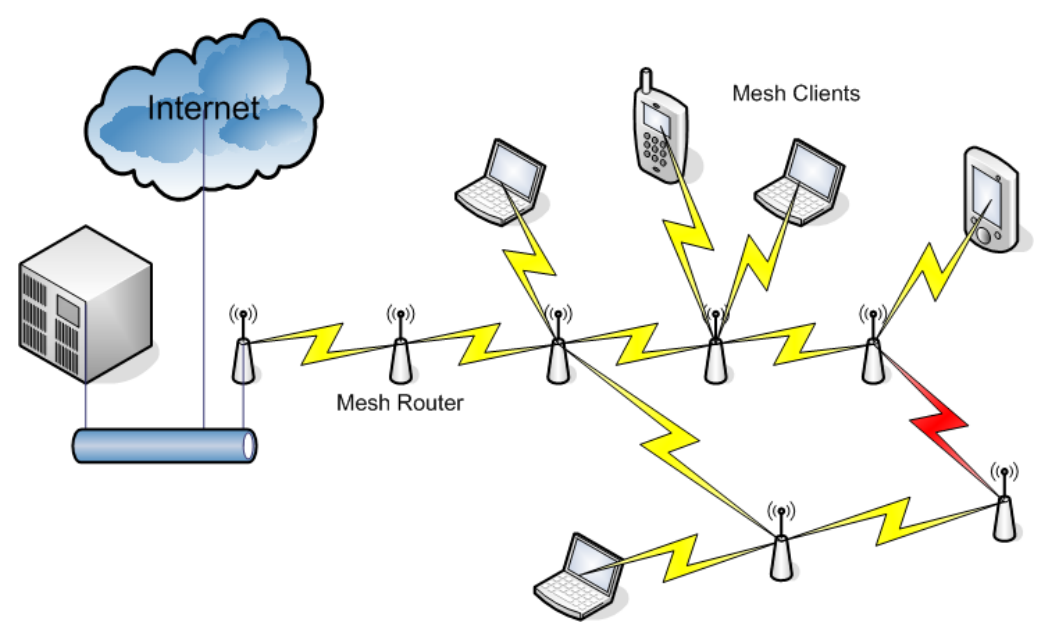

Figure 2.3: A typical wireless mesh network configuration.

One architecture type of WMNs is the infrastructure or access WMN. In this configuration, mesh routers are linked together wirelessly to form a backbone that data can be sent over. Wireless clients can connect to any of the mesh routers, usually the one with the strongest or best signal for the client, to have access to the network. 
Clients with no wireless network interface can also connect via Ethernet to one of the mesh routers in order to gain access to the mesh network.

With WMNs, the number of hops refers to the number of mesh routers data must pass through. Traffic that passes through five hops is transmitted five times over the wireless medium between devices. Single hop traffic is transmitted only one time over the wireless medium. The location of a wireless station, the destination node, and the configuration of the WMN dictate the number of hops the data will pass through.

Another type of WMN is a backhaul network. On this kind of network, the wireless medium is only used to send data from hop to hop without having any mobile clients. This type of WMN could be used in a situation where two wired networks are to be connected together wirelessly, but the distance is too great for a single wireless hop.

WMNs allow for low cost extension and implementation of large networks. This type of network can be particularly useful for VoIP applications. As an example, low cost mobile telephone service could be provided with coverage over an entire site, office, or campus quite readily, therefore allowing users of the network to stay in touch while moving throughout the coverage area.

\subsection{Related Performance Works}

Performance of voice in 802.11 networks was studied in several papers. In [3], voice performance on a single hop wireless network was studied, however, in 2003, the 802.11e standard had not yet been ratified, so QoS could not be studied. In [13], throughput of UDP traffic and voice performance was studied. In this case, testing was also done on a single hop wireless network. In [26], experimental tuning of 802.11e parameters was conducted, but only on a single hop network. In [18], a 
simulation was performed to study the dynamic tuning of the CWmax parameter. In [28], performance in $802.11 \mathrm{WMNs}$ was studied with respect to voice, but no QoS implementation was used.

In [3], a testbed network was configured to test 802.11 single hop wireless network performance. Anjum, Dutta, et al measured the performance and capacity of the standard 802.11 DCF mode to deliver high quality voice service with and without background traffic.

In [13], performance of UDP traffic and VoIP was studied over a single hop 802.11b network, from the perspective of the number of simultaneous calls that a single access point can handle. Garg and Kappes discuss that due to the small payload size of the voice packets, the 802.11 network becomes inefficient and cannot support many calls. The study found that only six calls could be supported on an access point when the G.711 CODEC was used with a $10 \mathrm{~ms}$ sampling time. This equates to 100 packets per second. We expect to be able to handle more simultaneous calls with the more normal $20 \mathrm{~ms}$ sampling time.

In [26], experimental tuning of the CWmin parameter is tested in a single hop configuration with respect to voice calls. Davis and Narbutt test numerous CWmin parameter settings and determine that adjusting the CWmin of the background traffic has a positive effect on the voice call quality.

In [18], dynamic tuning of the CWmax parameter was carried out in a simulation. Hanley and Murphy showed that an adaptive scheme in a simulation environment could increase performance on a single hop network. Results show that the adaptive scheme that dynamically adjusts the CWmax parameter can indeed increase performance and can consistently provide a higher VoIP call capacity. 
In [28], an experimental mesh network testbed was configured to test the performance of VoIP. Niculescu et al tested several configurations, including a standard single radio setup and multiple radio setup. They also tested packet aggregation. The standard single radio configuration tested the number of calls that can be supported at one time.

The above papers do not all cover mesh networks, nor do they cover the three combined features that we cover in our research. These are, WMNs, VoIP, and QoS. We are testing how well the current QoS mechanism in 802.11 works with respect to VoIP, background traffic, and WMNs. These, to our knowledge, have not been previously combined. The experimental value of the other works applies to specific configurations only. None of the above papers study WMNs, VoIP, and 802.11e QoS simultaneously, so it is difficult to use their experimental results to compare with ours.

In addition to the above mentioned papers, there are several others that deal with modification to the 802.11 standard, and as a result, are not implemented on any commercially available 802.11 routers.

Two schemes discussed in [39] deal with packet multiplexing and header compression. Packet multiplexing is the process of analyzing packets at each node and merging packets together temporarily. Only packets that are travelling in the same direction and on the same link can be multiplexed. Header compression is a process that takes place at each node and compresses the IP, UDP and RTP header down in size, therefore increasing the wireless network payload efficiency of the transmission.

The proposed schemes in [39] demonstrate through simulation that multiplexing can improve the VoIP capacity, but must be implemented at the access point and 
requires significantly more processing power than most access points currently have. Header compression also provides a slight performance increase, but also requires more processing power. Both schemes, due to the extra processing, increase delay, but are certainly an option worth exploring on high end access points. In [39], it is stated that 802.11e can provide a solution to the QoS problem, but tuning the parameters and evaluating the results is still an outstanding issue that needs to be investigated.

Gauging call quality from WLAN resource usage is discussed in [25]. Davis and Narbutt [25] shared that establishing a new call on a wireless network near capacity when calls are already active can dramatically decrease call quality. They proposed an application that passively monitors wireless local area network resource usage and can provide the information to a call admission control system. The purpose of the passive monitoring approach proposed is to allow a call to be established if there are enough available resources, while not allowing a call if the available resources are not sufficient.

We expect to see a similar decrease in call quality when we perform a test to determine the maximum number of calls that can be supported on our WMN testbed.

\subsection{Summary}

In this chapter, we provided an overview of the IEEE 802.11 protocol, and discussed the 802.11e QoS mechanisms. We also discussed WLANs, VoIP, metrics of VoIP, as well as SIP. In addition, we discussed WMNs and their uses, and included a brief discussion of some other performance studies relating to our work. No previous work has studied the effects of 802.11e QoS on a WMN with VoIP traffic. Our work 
combines these three features and studies many different scenarios for evaluating call quality. 


\section{Chapter 3}

\section{Implementation Details}

In this chapter, we provide implementation details of our experimental WMN testbed. We also describe the WMN configuration, the method for measuring performance metrics, and the method for processing the experimental results, as well as calculating call quality.

\subsection{Experimental Setup}

All data used in the performance evaluation chapter was collected during live experiments on an experimental mesh network testbed. The network is comprised of ten routers for the largest experiment, and as few as two for the smallest experiment.

In our experimental WMN testbed, the mesh routers used are single radio 802.11 routers. With the DD-WRT [16] software, these routers are versatile in what can be

done with them because of their Linux [22] software base. They can be configured as clients, access points, and network bridges, and have many other configuration options. The implementation of $802.11 \mathrm{e}$ and the ability to control many parameters 
of the routers allows us to test various experimental scenarios.

\subsubsection{The DD-WRT Software}

The routers used in the WMN testbed come from the factory with a small, flash memory sized version of Linux, custom built for use with home networks and broadband Internet connections. They provide wireless access, wired access, and address translation facilities to clients on the network. This software is maintained by the manufacturer, Linksys [21]. Since this software is designed for consumer use, many features that would normally be possible to use with a Linux based router are hidden or disabled.

To allow access to an extended featureset, open source software for these routers is being produced. This software, based on the original Linksys Linux firmware, is called DD-WRT ${ }^{1}$. The software can be specially loaded onto the routers in order to provide more configuration options and an extended featureset, particularly on the wireless interface, which is what we are interested in. This software uses a newer Linux kernel and provides a clean looking, web-based interface to adjust configuration parameters.

When DD-WRT is first flashed, the software creates a vanilla router, that is, a normal single radio 802.11 wireless router that gives the option of more advanced configuration. One such configurable option added to the routers are the 802.11e EDCA QoS features, allowing for configuration of QoS on the wireless interface.

We use DD-WRT because it allows us to test the 802.11e QoS provisions on a WMN. DD-WRT also provides access to the settings through the web-based interface. In addition, it facilitates the implementation of the WMN testbed by providing open

\footnotetext{
${ }^{1}$ DD-WRT is not an acronym. DD is not defined. The letters WRT are chosen from the model name of some Linksys routers.
} 
source ready to go software.

The reader can refer to Appendix A for more details on the DD-WRT software and screenshots of the configuration interface.

\subsubsection{Mesh Network Configuration}

The generic configuration of our WMN testbed (Figure 3.1 on page 29) is a forced linear configuration, with each router forwarding packets on to the next in a linear pattern. This allows for a defined, specific test configuration that will not change during an experiment. This contrasts to a WMN with dynamic routing, where the next hop is dynamically chosen based on link quality or other factors. This configuration is typical of traffic in a mesh network, with traffic flowing across the network from a computer to a gateway node. The traffic may traverse only one hop, or it may traverse multiple hops. The number of hops that traffic will traverse depends on how far away the wireless station is from the gateway node and the configuration of the mesh network.

The DD-WRT software supports a network mode called Wireless Distribution System (WDS). This mode configures the mesh router as a network bridge and access point simultaneously. That is, it will transparently act as a bridge on the network and also allow mesh clients to associate with it. The problem with this mode is that the 802.11e QoS functionality does not operate in WDS mode. To overcome this difficulty, the mesh routers are configured in Client Bridge mode. In this mode, the mesh routers act as clients of one another, but still provide access point functionality, while permitting the use of the 802.11e QoS functionality. 


\subsubsection{Basic Configuration Parameters of the Mesh Network}

Several 802.11 parameters were configured to specific values in order to obtain consistent results. These options include the transmit rate of the nodes, CTS protection mode, frame burst, transmit power and encryption settings, among others. A table of the non-QoS configuration parameters chosen is shown later, in Table 3.1. A discussion of the reasoning for the choices is offered below.

The transmit rate of an 802.11 device is normally controlled automatically based on channel conditions. Preliminary testing showed that automatic rate setting, with a maximum of $54 \mathrm{Mbps}$, resulted in wildly unpredictable performance and extreme error spikes. A fixed rate setting of $11 \mathrm{Mbps}$ provided a low error rate and consistent performance, while not being too slow to prohibit a voice call at a hop count of five on the mesh network.

The RTS and CTS mechanisms help to improve performance in 802.11 wireless networks as discussed in Subsection 2.1.1 on page 7. CTS protection mode prevents 802.11b clients from being blocked in an $802.11 \mathrm{~b} / \mathrm{g}$ environment where there are many $802.11 \mathrm{~g}$ clients competing for access to the medium. To offer this protection to $802.11 \mathrm{~b}$ clients, overall performance is decreased. Since the experimental testbed does not contain a large number of both $802.11 \mathrm{~b}$ and $802.11 \mathrm{~g}$ clients, this option has been disabled on the mesh network routers.

Frame burst is an option which offers increased performance when there are very few clients. The way that it works is by transmitting more packets than normal, therefore tying up the medium for a longer period of time. This makes other stations wait longer, which explains why it only works well if there are a very small number of clients. In a mesh network, where routers are retransmitting packets and there are 
also clients accessing the network, frame bursting decreases overall performance. As a result, frame bursting has been disabled on the mesh network routers.

The transmit power setting on mesh routers adjusts the amount that the signal should be boosted before being transmitted. On the mesh routers in our testbed, it is not a useful value to change. It may, however, be useful in a case where a mesh network is only a backhaul network. The problem with increasing the transmit power when there are mobile clients is that while the increase may permit a mobile client to better hear a node, it does not mean that the node can hear the mobile client any better. This is because the transmit power of mobile clients, such as a laptop, PDA, or smart phone, is not usually adjustable by the user. In our case, mesh clients can directly associate with any of the mesh nodes, so the transmit power setting was kept at the default of $28 \mathrm{~mW}$. This default was found to work well and provide good signal strength and integrity between mesh nodes.

Encryption is not used in the mesh network because of the additional overhead incurred by using encryption. We are not concerned with the ability of encryption or security for this work. The nodes are all running in clear, unencrypted mode to provide the best possible performance. With encryption enabled, we would expect to see a slight decrease in performance, both in total throughput and in call quality. 
Table 3.1: DD-WRT Mesh Router Settings

\begin{tabular}{|c|c|}
\hline Setting Name & Value \\
\hline Basic Settings / Wireless Mode & Client Bridge \\
\hline Adv. Settings / Basic Rate & Default \\
\hline Adv. Settings / Transmission Fixed Rate & 11 Mbps \\
\hline Adv. Settings / CTS Protection Mode & Disable \\
\hline Adv. Settings / Frame Burst & Disable \\
\hline Adv. Settings / WMM Support & Enable \\
\hline Adv. Settings / No-Acknowledgment & Disable \\
\hline
\end{tabular}

A general schematic of the mesh network described in this section can be found in Figure 3.1.

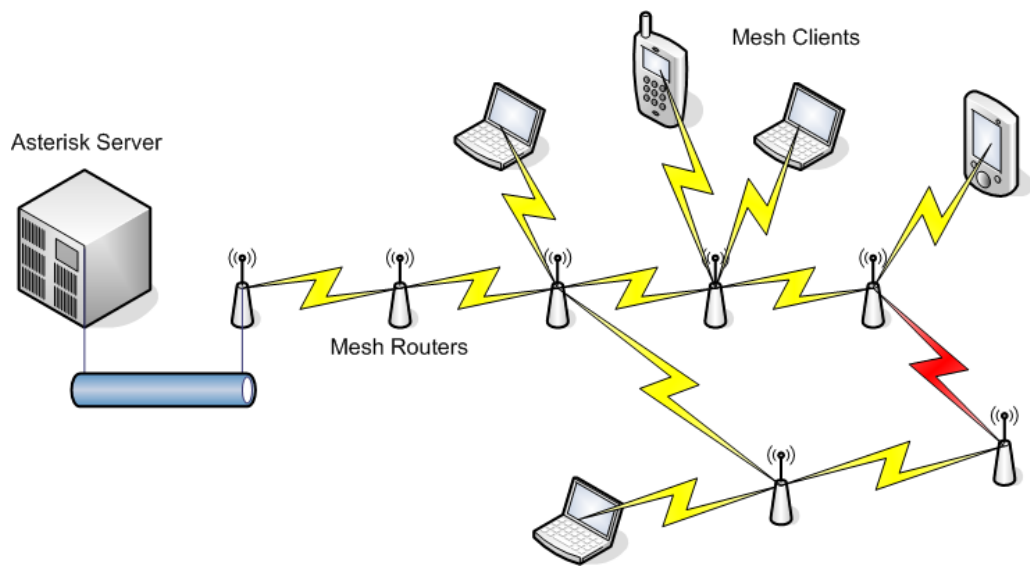

Figure 3.1: The general mesh network testbed configuration, as used in our research.

The mesh routers are placed in Goodwin Hall on the Queen's University campus and are equally spaced. Two routers are on the sixth floor, and one router is on each of the fifth, fourth, and third floors. A diagram depicting this configuration can be found in Figure 3.2. It was found that regardless of the spacing of the routers, call quality did not change significantly, unless routers were out of range of one another. 


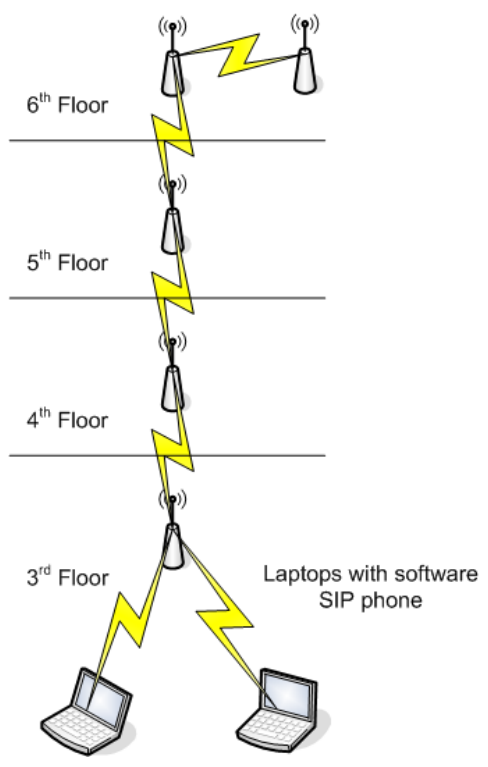

Figure 3.2: A diagram showing router placement in the building.

\subsubsection{Limitations of Using DD-WRT}

The use of DD-WRT, while allowing for a low cost WMN, does not allow for complete freedom in configuring the routers. First, DD-WRT does not support dual-radio mesh routers. The use of dual or multi-radio mesh routers would allow for further experimental testing in a wider range of scenarios. Many multi-radio mesh routers use a different protocol for the backhaul, that is, to send the traffic from router to router. These mesh routers use normal $802.11 \mathrm{~b} / \mathrm{g}$ for client access but can use 802.11a, WiMAX or microwave for the backhaul. Using a different network type for the backhaul would provide increased performance over the single-radio mesh routers we used, as the backhaul traffic would not interfere with client traffic.

Another limitation of using DD-WRT is that it does not allow us to inspect the amount of delay, jitter and packet loss introduced at each mesh network hop. Instead, we are able to capture this data at the call endpoints only. The only useful hop by 
hop data that we can see is the wireless interface error rate. This data was used in determining the fixed rate that the mesh nodes should be configured to transmit at. Regardless, DD-WRT is a good choice for us to use in our WMN.

\subsubsection{Asterisk}

To provide SIP and VoIP call termination over our WMN, we use Asterisk. Asterisk [11] is an open source telephony system that provides a wide range of functionality on many operating systems, including Linux. While Asterisk is open source, it is developed by Digium [19], a company that also sells hardware for Asterisk systems as well as turn-key solutions. The hardware Digium sells includes line cards for connecting Asterisk systems to existing telephone lines, digital T1 connection cards, and others. They offer ready-to-go Asterisk solutions that include AsteriskNOW and SwitchVox, and they also provide a business version of Asterisk that includes full support. So, while Asterisk is open source, there is motivation for it to be developed well. It is the natural choice for us to use because it runs on Linux and provides SIP registration, call routing and call termination services.

We also used it to perform real-world testing of voice calls and voice call termination over the Internet. The use of Asterisk provides valuable feedback for testing the network through the use of an echo test system. This is a system that repeats back what it hears, so that simple audible network testing can be done quickly and easily. It is also important to note that while we do use Asterisk in our research, it does not have any effect on call quality, as once an RTP stream is established between call endpoints, Asterisk does not interact with the stream.

Asterisk is a commonly used VoIP system. The Asterisk system can run at the 
core of an IP-based phone system or act as a gateway in a hybrid PBX system. It switches calls, manages call routes, acts as an Interactive Voice Response (IVR) system, handles conferencing, call parking, voicemail, unified messaging, among many other features. Asterisk provides the features we need for our work, including SIP support and VoIP call processing. Other options were explored, however, no other configurable open source PBX solution exists for us to use for our work. Figure 3.3 is a diagram of a typical Asterisk setup.

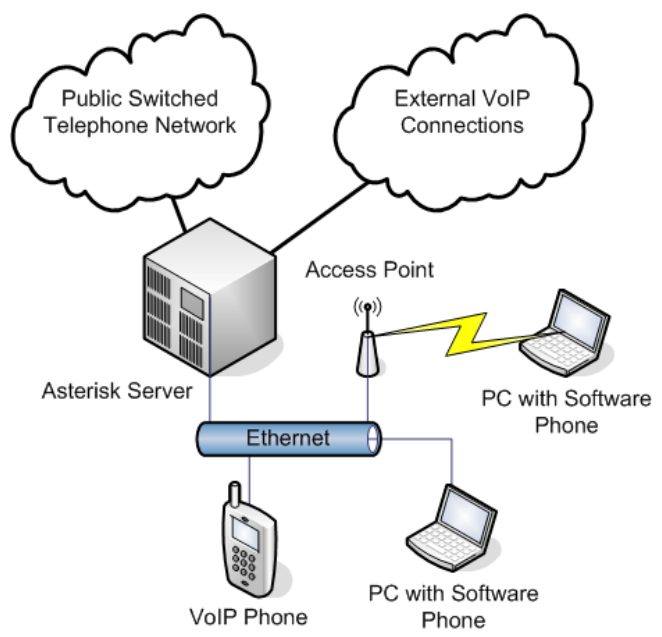

Figure 3.3: Diagram of an Asterisk setup.

\subsection{Measuring Call Performance}

To measure call performance, a value known as the MOS is used. This is an industry standard number, detailed in ITU-T recommendation P.800 [34] that represents perceived call quality and ranges from 1 to 5 . In large scale testing, this value is actually calculated using provided scores from many people listening to a call. The ITU introduced a method of calculating MOS using a formula called the E-model. 
MOS and E-model will be discussed in the next few sections.

\subsubsection{Mean Opinion Score}

In voice communications, and used primarily for VoIP and voice data that is compressed, the MOS is a representation of the quality of human speech. To determine MOS for a specific configuration, a number of listeners rate the quality of test sentences read by both male and female speakers. Each sentence is given a rating, from 1 to 5,1 being the worst, and 5 being the best. The MOS of a specific configuration is the arithmetic mean of the individual MOS values as recorded by the listeners. Typical MOS values of a cell phone call are in the upper-3 range while a land phone line ranks in the mid-4 range.

In our research, we are looking for a MOS value of 3.1 or greater. Below 3.1 is considered unacceptable quality and any call that has a MOS of less than 3.1 is not counted as a successful call [37].

MOS is a very useful means of measuring voice quality, as it allows for easy comparison of voice call quality from one test to the next. The drawback with MOS, however, is the cost of hiring many people to listen to sentences and rate them.

\subsubsection{E-model}

The E-model is a recommendation published by the ITU-T, given recommendation identifier G.107 [37]. It is a transmission rating model that gives an estimated call quality based on network factors. The most recent version of the E-model was approved by the ITU-T in the year 2005 [37]. 
We chose to use the E-model because it takes into account a wide range of impairments, such as CODEC choice, end-to-end delay, packet loss and jitter. Given these inputs, the E-model produces a rating factor, $\mathrm{R}$. This rating factor can be transformed to give an estimated MOS value, which we use for measuring call quality in our experiments. The minimum MOS value of 3.1 is equivalent to an R-value of 60 , which is the minimum call quality recommended by the ITU-T in [37].

One drawback of any call quality calculation such as the E-model is that it is only an estimate of sound quality. It does not test actual sound quality, but estimates it based on the performance metrics measured on the network. Other methods of calculating call quality are proprietary and not published or available without purchasing specific software. The formulae used to calculate the E-model R-value and conversion to MOS are detailed in Appendix B.

\subsection{Gathering, Processing, and Use of Experimen- tal Data}

\subsubsection{Gathering the Data}

When gathering the data, test scenarios were carried out a minimum of five times for a period of two minutes each. The first few seconds of each test were removed from the dataset. This is to allow the network conditions to stabilize and become steady state. The amount varied from one up to five seconds. None of the tests had unstabilized network conditions beyond five seconds.

The computers used for call tests run a software SIP phone called X-Lite [8]. This program allows two simultaneous calls per computer and also allows for configuration 
of the DSCP field for QoS queue insertion. It is free software and supports SIP, which is why we chose to use it. Figure 3.4 below shows a sample of the interface.

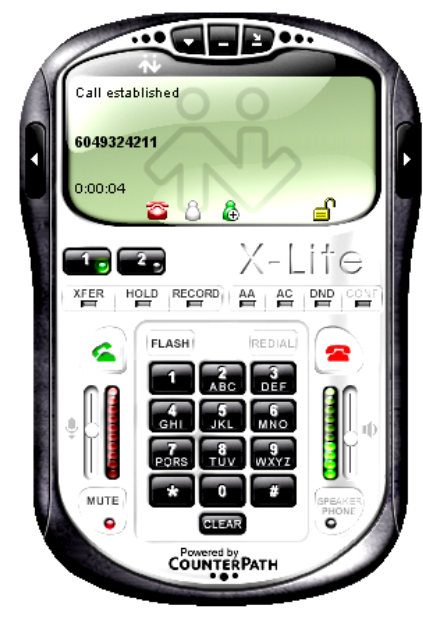

Figure 3.4: Example X-Lite software SIP phone interface.

The software used for capturing the data is called Wireshark [7], and collects data using the WinPcap [33] interface, which is a packet capture library for Windows. On the Linux system running Asterisk, data was captured using the dumpcap binary included with Wireshark. The resulting captures are then exported through Wireshark as Comma Separated Value (CSV) files for each direction of each call. The CSV files show packet send time, packet arrival time, sequence number, and packet number of the RTP stream. Wireshark was chosen because of its multi-platform usage and good interface. Figure 3.5 below shows a sample of the Wireshark interface with a live packet capture. 


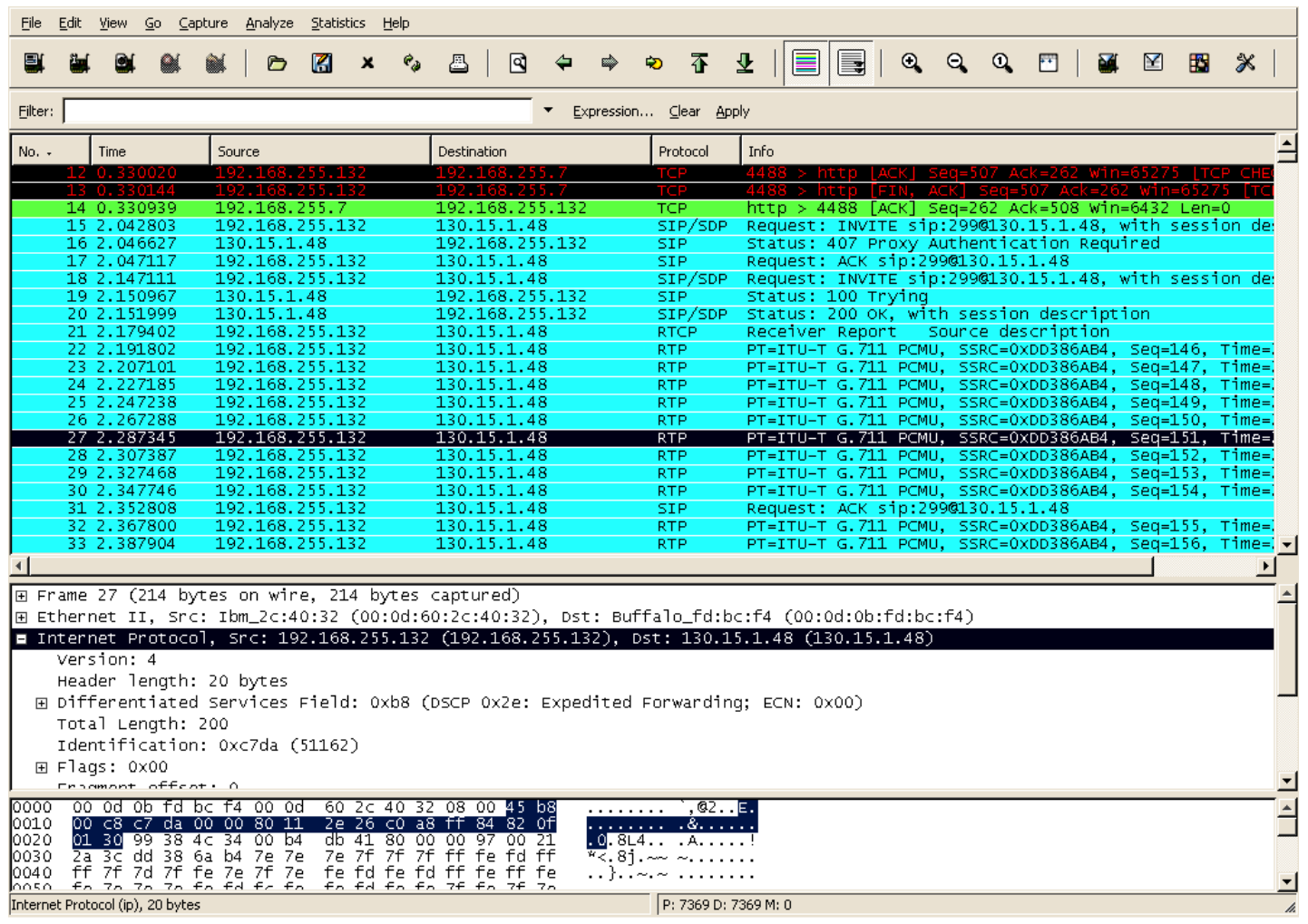

Figure 3.5: Example Wireshark interface with a live packet capture.

Background traffic, when required, is TCP traffic that utilizes the available bandwidth. This type of traffic simulates the traffic seen on most networks. We generate traffic using File Transfer Protocol (FTP) and HTTP by starting an FTP transfer and visiting an automatically reloading HTTP page.

\subsubsection{Processing the Data}

To process the data, we designed a program to process the CSV files. This program takes the packet send time, packet arrival time, sequence number, and packet number and calculates end-to-end delay, jitter and packet loss that are needed to calculate the R-value using the E-model. 
The program proceeds in the following manner:

- A data file is loaded into memory, ignoring the first few seconds of data.

- Several additional fields are calculated after loading the data.

1. The difference between arrival time and send time. This is the end-to-end delay of each packet.

2. The jitter value, by calculating and averaging the deviation from packet send time to packet arrival time.

3. The number of packets lost, by calculating the number of missing sequence numbers. Packet loss is expressed in percentage.

- After these calculations are made, an average end-to-end delay and jitter value can be calculated for a call. The average end-to-end delay is used because we need a single delay value for the E-model calculation, and is a good representation of actual end-to-end delay.

- The program then calculates the E-model R-value using the average end-to-end delay, jitter, and packet loss percentage.

- Finally, the program converts the R-value to MOS.

After the successful execution of the program, the MOS value for the call is stored in a table. Each direction of the call is stored separately, as are the individual test runs. For one call we end up with ten MOS values. 


\subsubsection{Use of Processed Data}

The ten MOS values for each call are eventually merged, first by averaging the different test runs of each direction together, then averaging the two directions together. The result is a single MOS value for a call in a specific configuration that can be used in our analysis, tables, and graphs.

Voice echo is not accounted for in the MOS calculation, as echo is an effect that is produced by the end devices and not the network. High delay on a network can increase the chance of echo, but echo cancellation functions on end devices should be able to remove echo without any issues.

\subsection{Summary}

In this chapter, we described the experimental testbed mesh network. We also described the generic configuration of the network, call quality information, as well as the gathering and processing of the experimental results.

In the next chapter, we present the test scenarios configured and used, along with the results from the different scenarios used. 


\section{Chapter 4}

\section{Performance Evaluation}

In this chapter, we explain the specifics of the individual scenarios and present the results. The network is configured as described in the previous chapter. We run each of the tests five times. Following the details of the scenarios, we summarize the results.

\subsection{Experimental Results}

In the experimental results section, we test many different scenarios and comparing the performance of the calls in each scenario. The types of tests we execute are as follows. We test the effect of increasing number of hops and background traffic on voice call quality. Following this, we test the effect on call quality as more calls are added to the network at different hop counts. This allows us to determine the maximum number of supported calls at different hop counts. Then, we test the fairness exercised by the network on calls in different configurations. After this, we test the amount of bandwidth that is available in other areas of the network when 
calls are active. This allows us to test what effect making calls in a certain area of the network has on other areas. Then, we test call quality of simultaneous calls with different QoS parameters. Finally, we test the effect that a single mesh router handling a large amount of traffic has on voice call quality and background traffic traversing it.

\subsubsection{Effect of Number of Hops and Background Traffic on VoIP}

The $\mathrm{n}$-Hops performance evaluation is meant to determine several important factors via a large set of tests. The objective is to determine test running values of the EDCA parameters CWmax and AIFS for the voice traffic class. To do this, we obtain call qualities, in the form of a MOS value, obtained from packet loss, jitter, and end-to-end delay in many different cases. The motivation of the n-Hops performance evaluation is to demonstrate the effects of increasing the number of hops in the network and modifying the EDCA parameters. In addition, the tests are performed with and without background traffic in order to show the effect of additional traffic on voice calls in different cases.

We use the CWmax and AIFS settings that are determined to give the good performance in the remainder of the tests in this chapter.

For this test, the WMN testbed is be configured in a linear topology with the number of mesh hops ranging from 0 to 4 hops. One additional hop is always be present as a result of the client connection to the network. As a result, the testing is done with the number of total wireless hops ranging from 1 to 5 hops. We begin testing with the default EDCA parameters and perform testing with one call and 
two calls, both with and without background traffic, for a total of four different call configurations. Table 2.1 on page 11 shows the default EDCA parameters. A diagram of the network configuration used for this test follows.

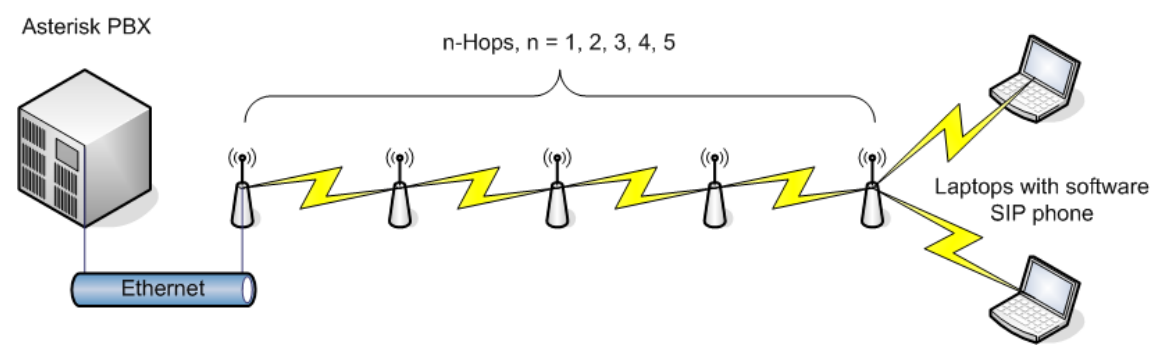

Figure 4.1: A diagram of the maximal 5 hop configuration used for the n-Hops performance test. The diagram shows the Asterisk PBX system, five access points, and two computers used as VoIP clients and background traffic endpoints. An identical configuration is used for varying the number of hops, but with fewer access points.

As shown in Figure 4.2, the voice call quality decreases with increased hop count. With no 802.11e QoS, that is, with all traffic having the same priority, call quality with background traffic drops off noticeably as hop count is increased. At one hop, call quality is good in all tests. When hop count is increased to two hops, the two call MOS with load drops below 4. This is because the background traffic has a greater effect on the voice call quality as the number of hops is increased. The quality of calls with background traffic decreases further with increased hop count while the calls with no background traffic remain roughly consistent in quality until we reach the five hop test. At five hops, even with no background traffic, call quality suffers with two simultaneous calls. This is because of increased packet loss due to retransmissions as well as increased jitter levels. The two call test with background traffic at five hops results in poor quality calls. We can make an early conclusion that only one call can 
be supported at five hops, and the introduction of a second call results in a call quality MOS of less than 3.1 for both calls, the minimum we are looking for.

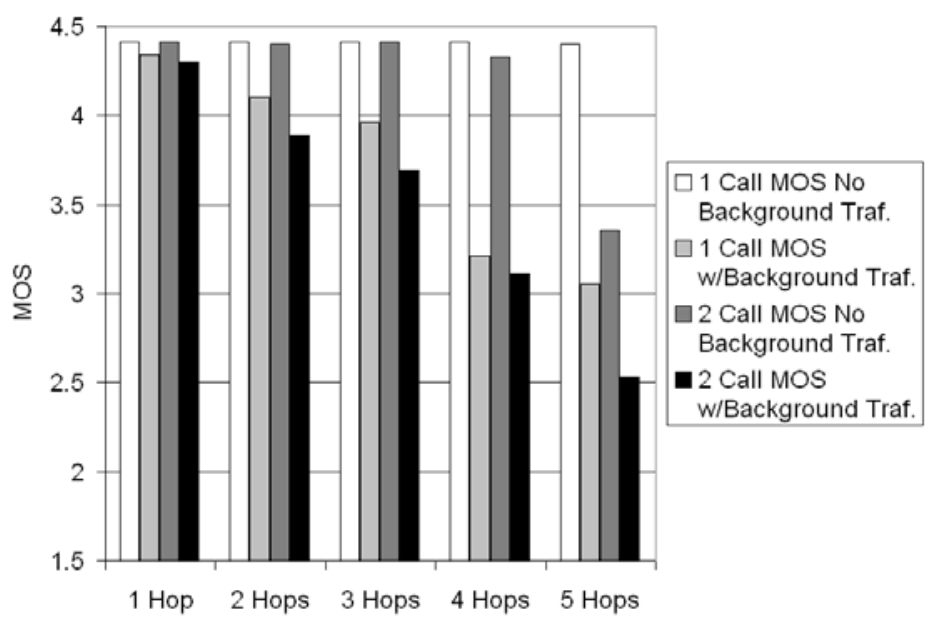

Figure 4.2: Graph showing MOS of 1 and 2 calls, with and without background traffic, with no traffic having priority over any other.

Increasing the CWmax parameter in the voice traffic class over multiple hops reduces the number of packets lost. While reducing the packet loss rate, it increases the average delay and therefore the average jitter as well. This is because voice traffic has the opportunity to use a larger contention window. For the next tests, we increase the CWmax value for the voice class from the default of 7 to $15,31,63,127,255,511$ and $1023 \mathrm{~ms}$. This allows us to settle on a CWmax value for the voice class that is an acceptable trade-off between number of packets lost and increased delay. 


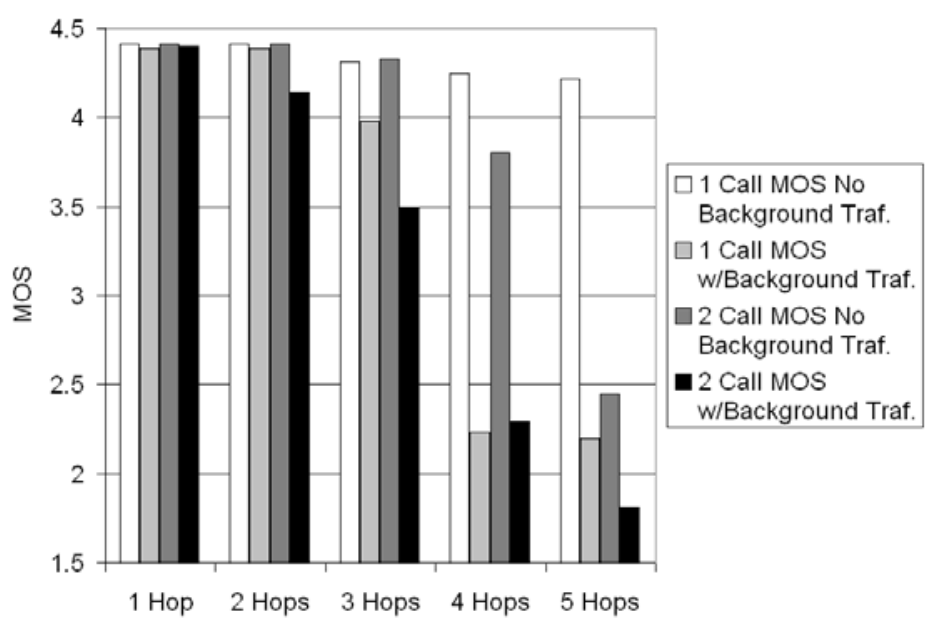

Figure 4.3: Graph showing MOS of 1 and 2 calls, with and without background traffic, with the voice traffic class having a CWmax setting of $7 \mathrm{~ms}$.

When voice traffic and background traffic are separated into their respective traffic classes (Figure 4.3), the performance of the voice call changes when compared to the case in which there is no 802.11e QoS and all traffic is in the same traffic class (Figure 4.2). With the default EDCA settings, in Table 2.1 on page 11, voice call quality is improved for the one hop and two hop cases in all call configurations. In the three hop case, call quality is similar, while in the four and five hop cases, call quality suffers, particularly in the cases with background traffic. This is because the voice traffic class has a CWmax of $7 \mathrm{~ms}$ in this test. With the maximum contention window length of $7 \mathrm{~ms}$, an increasing number of hops results in many more retransmissions and high packet loss rates. The average MOS of all calls when 802.11e QoS is disabled is 3.81 , compared to a MOS of 3.72 when the CWmax is at $7 \mathrm{~ms}$. This variation is seen because of the increased number of retransmissions and higher packet loss rate when the CWmax is at $7 \mathrm{~ms}$ for extended numbers of hops. 


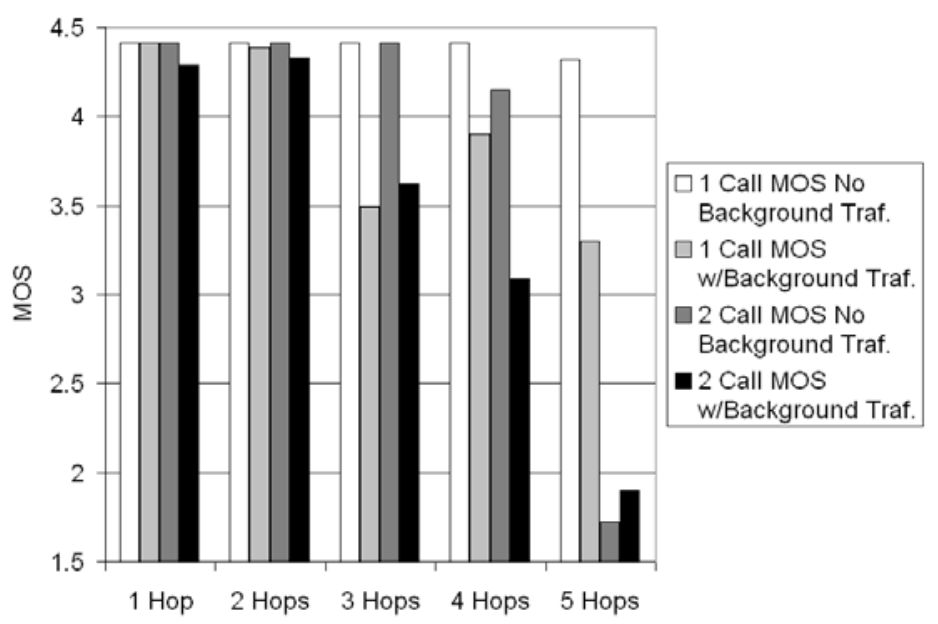

Figure 4.4: Graph showing MOS of 1 and 2 calls, with and without background traffic, with the voice traffic class having a CWmax setting of $15 \mathrm{~ms}$.

When the CWmax of the voice traffic class is increased to $15 \mathrm{~ms}$, an improvement is seen in both the four and five hop cases, as seen when comparing Figures 4.3 on the previous page and 4.4. The increase of the CWmax parameter gives the voice traffic a better chance of being transmitted successfully across an increasing number of hops. In particular, one call with background traffic at both four and five hops improves significantly, from 2.23 to 3.9 and 2.2 to 3.3 , respectively. The two call case with background traffic at four hops also shows a significant increase in voice call quality, from 2.29 to 3.09 .

With the CWmax of the voice traffic class increased to $31 \mathrm{~ms}$, the main change seen is in the two call five hop configuration, both with and without background traffic. Again, this occurs because the voice traffic has an increased chance to be transmitted across a high number of hops with a larger maximum contention window length. Call quality significantly improves, from 1.72 to 2.89 and 1.9 to 3 , respectively. When the CWmax of the voice traffic class is increased to $63 \mathrm{~ms}$ (Figure 4.5 below), call quality 
increases on average, from 3.97 to 4.04, but the two call five hop configuration does not improve further.

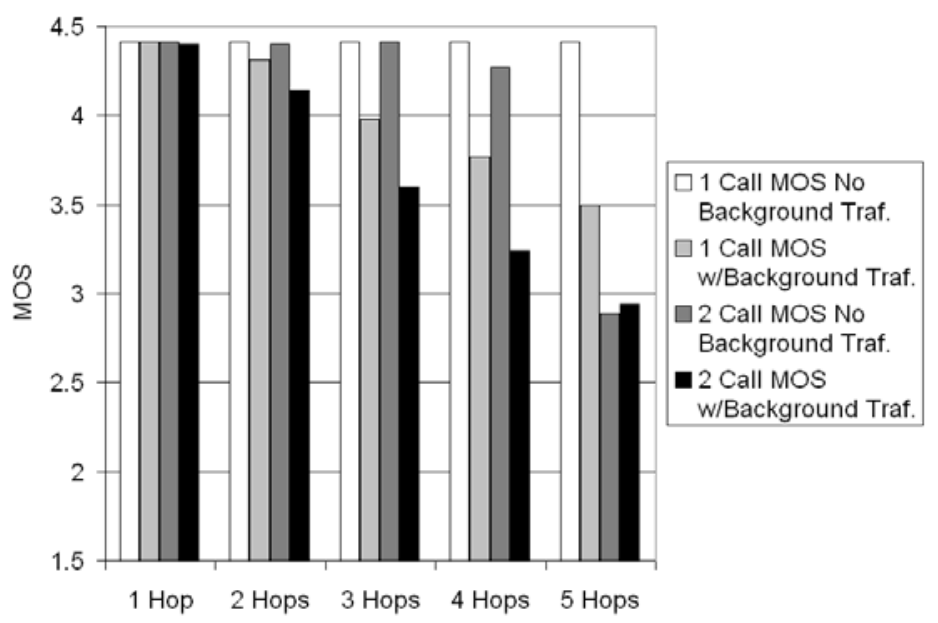

Figure 4.5: Graph showing MOS of 1 and 2 calls, with and without background traffic, with the voice traffic class having a CWmax setting of $63 \mathrm{~ms}$.

Further changes in the CWmax setting of the voice traffic class result only in minor changes to call quality. While increasing the CWmax of the voice traffic class does decrease the packet loss rate, it increases the average delay as well as the jitter. This effect of increasing the CWmax setting results in very small changes to the call quality beyond the $63 \mathrm{~ms}$ setting, all the way to the maximum of $1023 \mathrm{~ms}$. The decreased packet loss rate offsets the increase to average delay and jitter, and keeps the net gain in call quality near zero.

The figure below shows the call quality information for the CWmax setting of $1023 \mathrm{~ms}$. When compared to the CWmax setting of $63 \mathrm{~ms}$, the average MOS drops from 4.04 to 3.96 . 


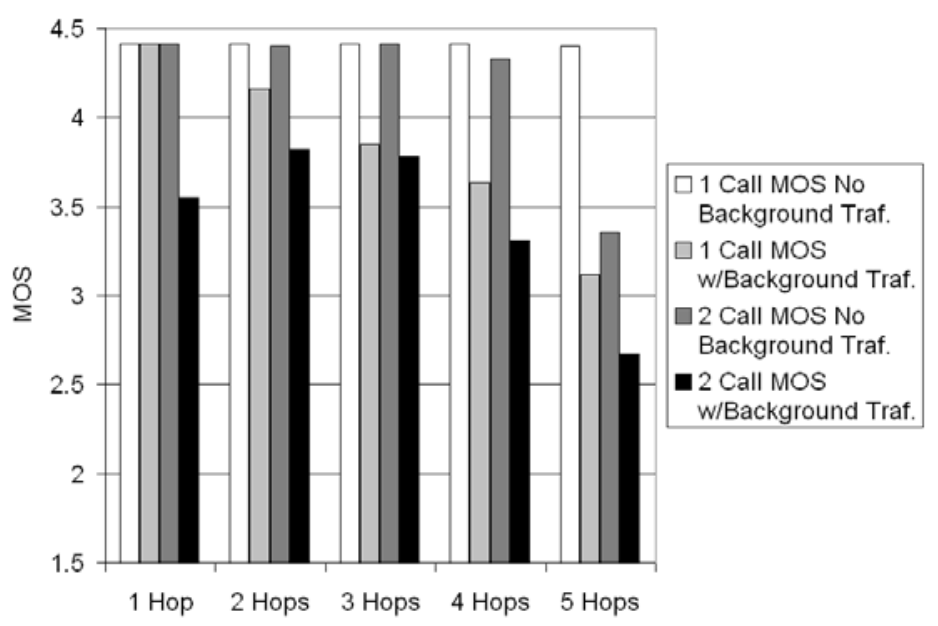

Figure 4.6: Graph showing MOS of 1 and 2 calls, with and without background traffic, with the voice traffic class having a CWmax setting of $1023 \mathrm{~ms}$.

The result that gives the best overall call quality is obtained by setting the CWmax of the voice traffic class to $63 \mathrm{~ms}$. Since this setting gives the best overall call quality, it is the CWmax setting that we have chosen for all of the next tests. Table 4.1 summarizes the average call quality MOS values for each CWmax setting. The table summarizes the results from the charts above, as well as the data from the charts not shown (31 ms, $127 \mathrm{~ms}, 255 \mathrm{~ms}$ and $511 \mathrm{~ms}$ ). As shown, the CWmax of $63 \mathrm{~ms}$ results in the highest average MOS value. The higher CWmax value allows for lower contention on the wireless medium than the default CWmax value without causing excessive delays for voice traffic. 
Table 4.1: Average Call Qualities with Different CWmax Settings

\begin{tabular}{|c|c|}
\hline CWmax & Average MOS \\
\hline No $802.11 \mathrm{e}$ & 3.81 \\
\hline $7 \mathrm{~ms}$ & 3.72 \\
\hline $15 \mathrm{~ms}$ & 3.88 \\
\hline $31 \mathrm{~ms}$ & 3.96 \\
\hline $63 \mathrm{~ms}$ & 4.04 \\
\hline $127 \mathrm{~ms}$ & 3.87 \\
\hline $255 \mathrm{~ms}$ & 3.96 \\
\hline $511 \mathrm{~ms}$ & 3.96 \\
\hline $1023 \mathrm{~ms}$ & 3.96 \\
\hline
\end{tabular}

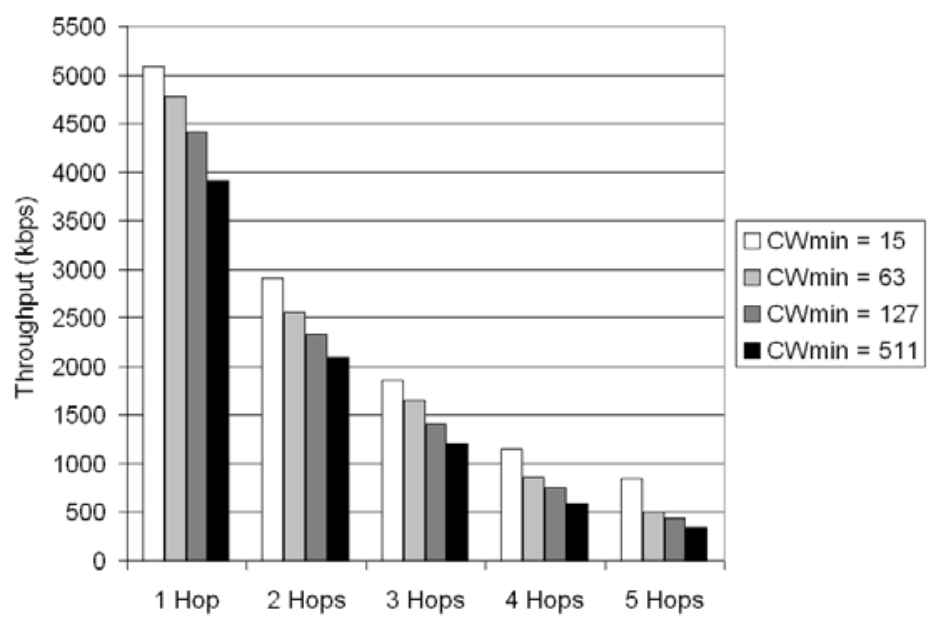

Figure 4.7: Graph showing the total throughput of background traffic at each hop count, first, with the default CWmin setting of $15 \mathrm{~ms}$, then with increased CWmin settings.

To lessen the effect of background traffic on voice traffic, we increase the CWmin of the best effort traffic. With a larger CWmin value, the best effort traffic is less likely to be transmitted at any given time when voice traffic is also waiting to be transmitted. The only downside to increasing the CWmin value is that the throughput of best 
effort traffic is reduced as the CWmin value is increased, even when there is no voice traffic present. This is not an ideal way to decrease the effect of background traffic on voice traffic, but is worth discussing as it seems an obvious way to make voice traffic perform better. Figure 4.7 above shows a drop in total throughput of background traffic for each increase to the CWmin setting.

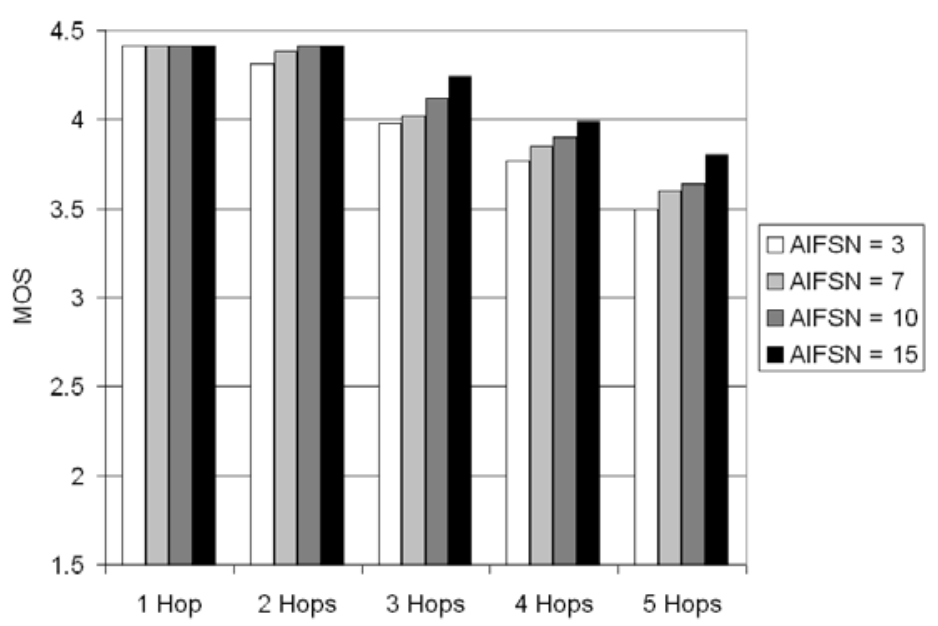

Figure 4.8: Graph showing the call quality with increased background traffic AIFS values.

An alternative to increasing the best effort traffic CWmin is to increase the AIFS value. This value represents the amount of time background traffic waits after the wireless medium is free before contending for the medium. The AIFS value of the the best effort traffic class is adjusted from the default of $3 \mathrm{~ms}$ to the values of $7 \mathrm{~ms}$, $10 \mathrm{~ms}$ and $15 \mathrm{~ms}$. Figure 4.8 shows call quality of calls with background traffic and the effect of changing the AIFS value of the background traffic on voice quality. As can be seen, call quality marginally increases with slightly higher AIFS settings. If the AIFS value is increased further, degradation in the throughput of the background 
traffic is seen, while the effect of background traffic on call quality does not change noticeably.

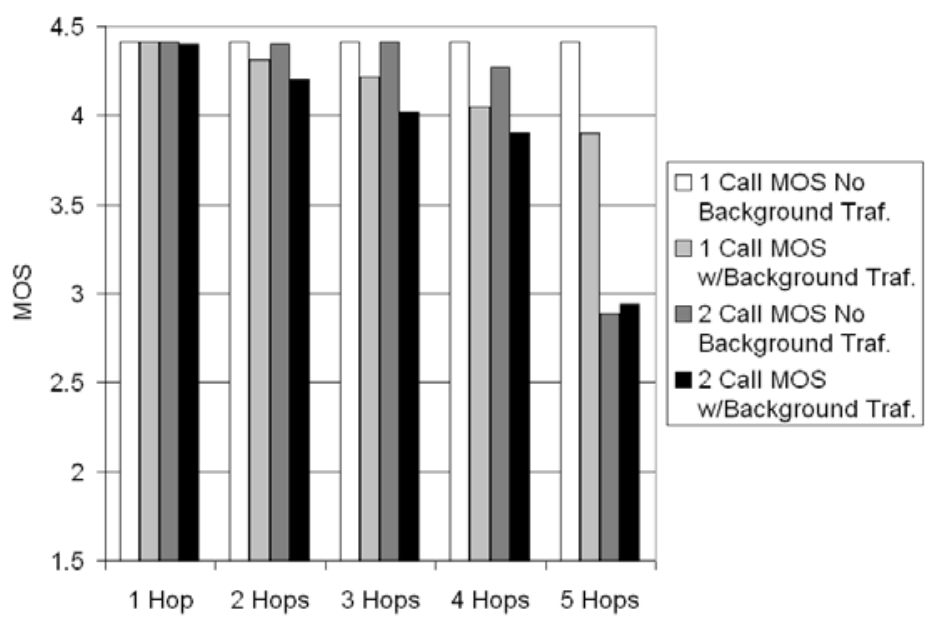

Figure 4.9: Graph showing MOS of 1 and 2 calls, with and without background traffic, with the voice traffic class having a CWmax setting of $63 \mathrm{~ms}$ and the background traffic class having an AIFS setting of $15 \mathrm{~ms}$.

The best parameter settings are a voice CWmax of $63 \mathrm{~ms}$ and an AIFS for the background traffic of $15 \mathrm{~ms}$. Using these parameters increase the call quality over multiple hops and decrease the effect background traffic has on call quality. In addition, this is done without severely impacting the background traffic even if it is the only traffic on the network at a given time. Figure 4.9 shows improvement in the call quality over the test done without increasing the AIFS value. Comparing the average call quality of all calls in this configuration with those calls in the test done without increasing the AIFS value shows an increase. These values are summarized in Table 4.2. 
Table 4.2: Average Call Quality Comparison of $63 \mathrm{~ms}$ CWmax with and without AIFS Adjustment

\begin{tabular}{|c|c|}
\hline CWmax, AIFS & Average MOS \\
\hline $7 \mathrm{~ms}$ (default), $3 \mathrm{~ms}$ & 3.72 \\
\hline $63 \mathrm{~ms}, 3 \mathrm{~ms}$ & 4.04 \\
\hline $63 \mathrm{~ms}, 15 \mathrm{~ms}$ & 4.14 \\
\hline
\end{tabular}

Using a CWmax of $63 \mathrm{~ms}$ for the voice traffic class and an AIFS of $15 \mathrm{~ms}$ for the background traffic class results in better performance than the defaults. These parameters are used in all of the next tests.

In summary, with our chosen settings of $63 \mathrm{~ms}$ for the CWmax and $15 \mathrm{~ms}$ for the AIFS, we have a $0 \%$ drop from the maximum MOS of 4.41 in call quality for a single call ranging from 1 to 5 hops. The MOS does not drop at all. When this is extended to include background traffic, we see a $2 \%$ drop in call quality from one to two hops. We see a further $2 \%$ drop in call quality from two to three hops, a $4 \%$ drop in call quality from three to four hops, and a $4 \%$ drop in call quality from four to five hops. This is a total drop in call quality of $12 \%$ from one hop to five hops.

When the number of calls is increased to two with no background traffic, the change from one hop to two hops and two to three hops is not noticable, with a $0 \%$ drop. The change from three hops to four hops shows a $3 \%$ drop in call quality. When this is extended to five hops, the drop in call quality from four to five hops is $32 \%$. This suggests that two calls at five hops is not sustainable on the mesh network testbed. This is a total drop in call quality of $34 \%$ from one hop to five hops versus a total of $3 \%$ from one to four hops.

When background traffic is included, the drops between hops are more significant. 
A 5\% drop is seen between one and two hops, a 4\% drop between two and three hops, a $3 \%$ drop between three and four hops, and a $25 \%$ drop between four and five hops. This is a total drop in call quality of $33 \%$ from one to five hops versus a total of $11 \%$ from one to four hops. Again, two calls at five hops are not sustainable.

\subsubsection{Maximum Number of Supported Calls}

The objective of the maximum supported calls test is to determine the maximum number of voice calls that can be supported at different numbers of wireless hops. The motivation of this test is to be able to define numbers that represent the maximum number of voice calls supported depending on how many wireless links are traversed in the test bed WMN. These numbers are useful for planning and deploying an actual WMN.

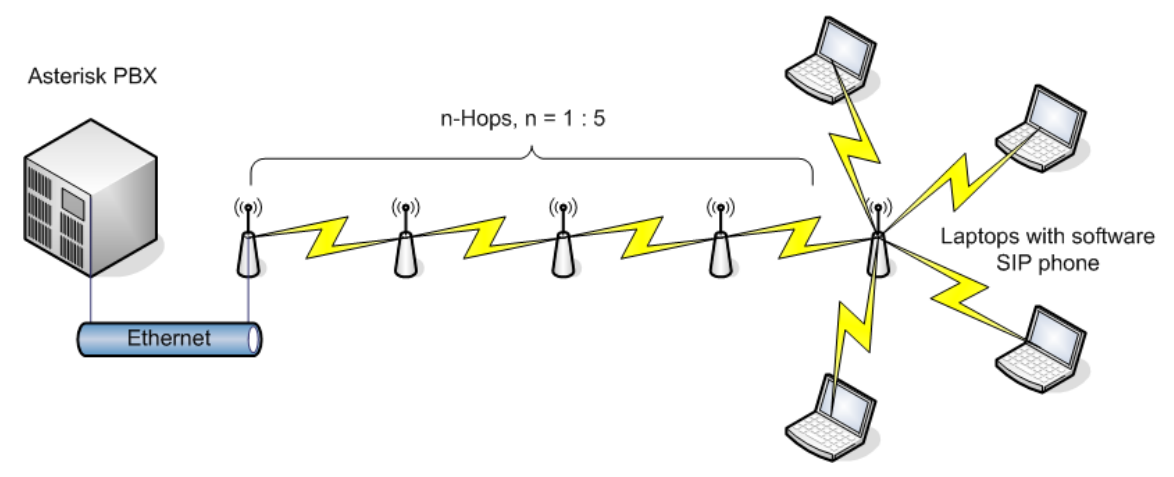

Figure 4.10: 5 Hop Network Configuration for Maximum Supported Calls Tests. For fewer hops, an identical configuration is used except for removal of horizontal wireless hops and access points. A maximum of 2 calls per computer endpoint are established.

Figure 4.10 shows the configuration for running the maximum supported calls tests. Four computer endpoints were used, all wirelessly connected to an access 
point. The number of wireless hops which traffic traversed varied from one to five hops. Each computer endpoint could run at most two simultaneous calls. For the one hop test, a fifth computer was added to allow the call limit to be found.

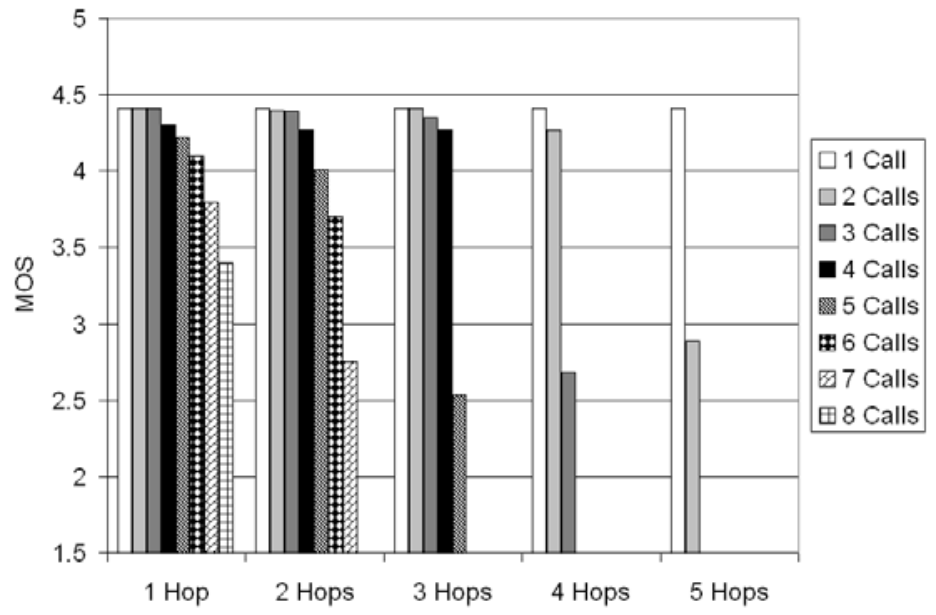

Figure 4.11: Graph showing MOS of 1 to 8 calls, if applicable, from 1 hop to 5 hops count.

As shown in Figure 4.11, the maximum number of supported calls decreases for each additional hop. At one hop, eight calls can be handled with the average MOS maintained above 3.1. A ninth call, added with a fifth computer, reduced the average MOS for the one hop test to 2.7. With calls traversing two hops, the number of calls that can be supported drops to six, as with seven active calls, the average MOS drops below 3.1. With three hops, the number of calls that can be supported drops to four calls, and with four hops only two calls can be supported. When the network is extended to five hops, only one call can be supported before the average MOS drops below 3.1. When additional calls are added in the two, three, four, and five hop cases, the average MOS drops below 1.5, which is why they are not shown on the graph. 
Fairness of the calls in these tests are discussed in the following section.

Recall that in Figure 4.7 on page 47, maximum actual throughput of the one hop case is approximately $5100 \mathrm{kbps}$ when running at a rate of 11 Mbps. Table 4.3 shows how much bandwidth is left available for background traffic after calls are added to the network. From these numbers, we can average the differences, and use the resulting number, $630 \mathrm{kbps}$, as the amount of bandwidth a call takes up over one hop.

Table 4.3: Bandwidth Available for Background Traffic With Calls Added to the Network

\begin{tabular}{|c|c|}
\hline Number of Calls & Bandwidth Available at One Hop \\
\hline 0 & $5100 \mathrm{kbps}$ \\
\hline 1 & $4485 \mathrm{kbps}$ \\
\hline 2 & $3932 \mathrm{kbps}$ \\
\hline 3 & $3301 \mathrm{kbps}$ \\
\hline 4 & $2656 \mathrm{kbps}$ \\
\hline 5 & $1994 \mathrm{kbps}$ \\
\hline 6 & $1302 \mathrm{kbps}$ \\
\hline 7 & $605 \mathrm{kbps}$ \\
\hline 8 & $59 \mathrm{kbps}$ \\
\hline
\end{tabular}

Even though a voice call using the G.711 CODEC only produces a 64 kbps voice stream for each direction, totaling $128 \mathrm{kbps}$, the actual effective usage on the wireless network is approximately $630 \mathrm{kbps}$ for the call to traverse one hop. This is because of the low efficiency on WLANs when transmitting small packets. The payload efficiency is approximately $20 \%$. The remaining $80 \%$ is taken up by the headers of the RTP, UDP, IP and MAC layers. These results are the same regardless of 802.11e parameter settings because the same amount of data is still being put on the network.

If we were to use a different CODEC, the total bandwidth used would change, but 
not dramatically. For example, using the G.729 codec would result in an 8 kbps stream for each direction, totaling $16 \mathrm{kbps}$. If the CODECs transmit at the same interval, once every $20 \mathrm{~ms}$, the total bandwidth drop would only be the difference between 128 kbps and 16 kbps, giving approximately 518 kbps actual effective bandwidth usage. This is due to the low payload efficiency of each packet. The large overhead when transmitting a small packet is one inherent problem with VoIP over wireless networks.

For this test, we considered the number of calls that can be supported at each hop. At one hop, eight simultaneous calls can be supported. At two hops, the number of supportable calls drops to six, a $25 \%$ drop. When the network is extended to three hops, four simultaneous calls can be supported, a further $33 \%$ drop. At four hops, two simultaneous calls can be supported, and at five hops only one simultaneous call can be supported. The fifth hop data supports the data from the first test showing a large drop in quality when two calls are active at five hops. They both show that only one call can be supported with the minimum quality level (MOS 3.1) maintained.

\subsubsection{Fairness of Calls at Different Hop Counts}

The objective of testing fairness between voice calls is to determine if the network maintains fairness between calls with the addition of the 802.11e QoS mechanism. We test if the network maintains fairness as varying numbers of calls are active on the network, active in different parts of the network. In addition, we test the effect of background traffic on the fairness between calls.

For example, two similar or identical calls should exhibit the same performance on the network in ideal conditions. The testing is done with the QoS settings of CWmax and AIFS as defined earlier. The configurations tested are chosen to show 
if the fairness is affected when calls begin to compete with one another for access to the wireless medium.

The fairness index used is the Min-Max fairness index [10]. This index is sensitive to degradation in call quality. A result of 1 means that the network treats the calls fairly, while less than 1 means less than fair allocation of resources. Fairness is measured between the lowest MOS value of a test set, and the highest MOS value of a test set. To calculate the Min-Max fairness index, the below formula is used:

$$
\text { Min-Max fairness index }=\frac{x_{\min }}{x_{\max }}
$$

where $x_{\min }$ is the lowest MOS value of a test set

and $x_{\max }$ is the highest MOS value of a test set

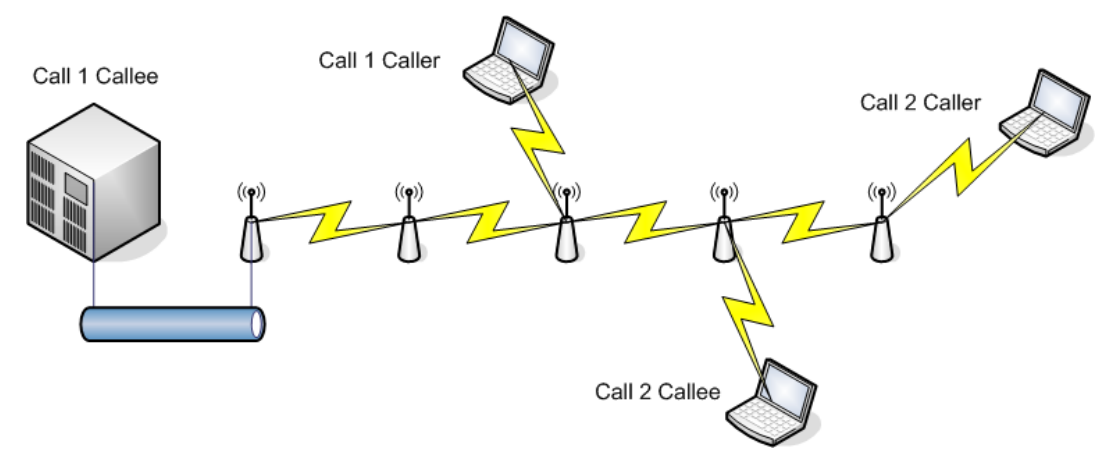

Figure 4.12: Diagram showing the configuration of the network for a fairness test with two calls on two separate portions of the network.

The first test, as shown in Figure 4.12, compares a call on one side of the mesh network to a call on the other side of the mesh network. With only one wireless hop in between them, some interference is expected. This test determines if this interference 
affects the performance of the calls at all. Ideally, the calls should exhibit the same performance. Table 4.4 shows the results of this test.

Table 4.4: Results of Fairness Test of Calls on Separate Portions of the Network

\begin{tabular}{|c|c|c|}
\hline & MOS of Call 1 & MOS of Call 2 \\
\hline Call 1 Alone & 4.41 & - \\
\hline Call 2 Alone & - & 4.41 \\
\hline Both Calls Simultaneously & 4.35 & 4.35 \\
\hline
\end{tabular}

The network treats the calls fairly, resulting in equal performance. With one hop spaced between the calls, interference does not effect call quality noticeably, though it does impact it slightly, as can be seen by the decrease in the MOS to 4.35. This is the expected result. The Min-Max fairness index gives a result of 1.00 .

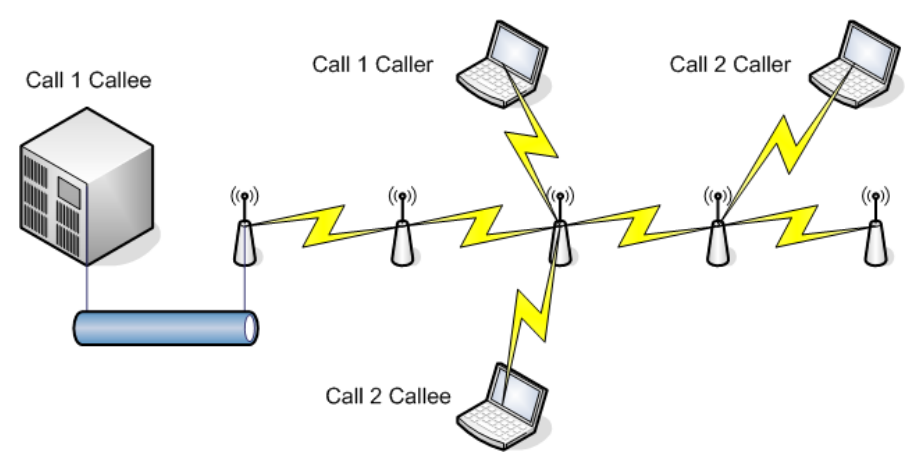

Figure 4.13: Diagram showing the configuration of the network for a fairness test with two calls on two separate but touching portions of the network.

The second test, as shown in Figure 4.13 compares a call on one side of the mesh network to a call on a separate part of the network, but with the two calls both having endpoints at the same node. We expect to see slightly degraded performance 
when both calls are active simultaneously, but the network should maintain fairness between the two calls. Table 4.5 shows the results of this test.

Table 4.5: Results of Fairness Test of Calls on Separate but Touching Portions of the Network

\begin{tabular}{|c|c|c|}
\hline & MOS of Call 1 & MOS of Call 2 \\
\hline Call 1 Alone & 4.41 & - \\
\hline Call 2 Alone & - & 4.41 \\
\hline Both Calls Active Simultaneously & 4.32 & 4.32 \\
\hline
\end{tabular}

Again, the network maintains fairness of the two calls, but additional use of the centre node reduces performance slightly when compared to the previous test with one hop in between calls. We expect to see the MOS slightly lower than in the previous test, and we do, with the MOS at 4.32 instead of 4.35. The Min-Max fairness index gives a result of 1.00 .

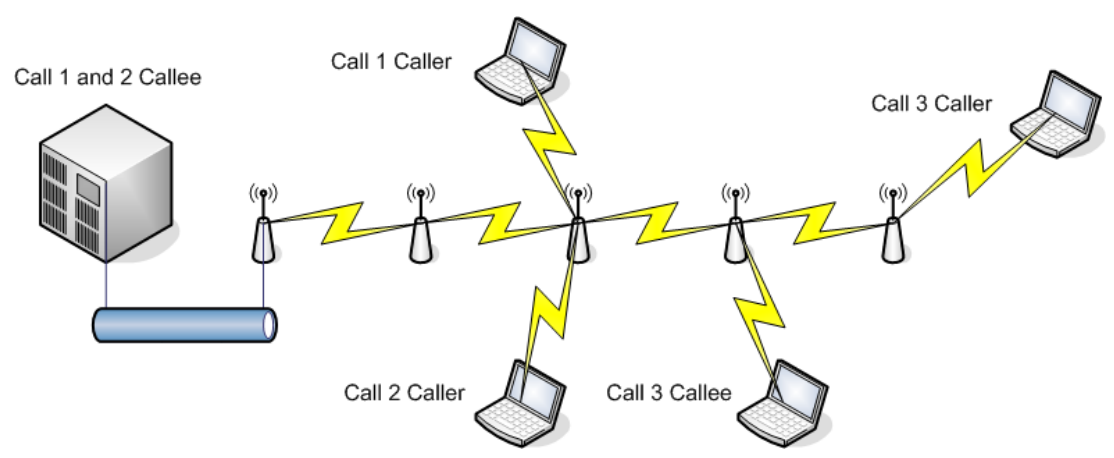

Figure 4.14: Diagram showing the configuration of the network for a fairness test with three calls: two overlapping, and one on a separate but touching portion of the network.

The third test, as shown in Figure 4.14, is identical to the previous test except an additional, identical call is added to overlap with Call 1. In this case, Call 1 and Call 
2 overlap on the mesh network, while Call 3 is adjacent to the other two calls. The expected result in this case is that Call 1 and Call 2 will have a lower MOS than Call 3 when all calls are active simultaneously due to the additional traffic on the portion of the network that Call 1 and Call 2 traverse. Table 4.6 shows the results of this test.

Table 4.6: Results of Fairness Test of Calls on Separate but Touching Portions of the Network

\begin{tabular}{|c|c|c|c|}
\hline & MOS of Call 1 & MOS of Call 2 & MOS of Call 3 \\
\hline Call 1 and 2 Simultaneously & 4.41 & 4.41 & - \\
\hline Call 3 Alone & - & - & 4.41 \\
\hline All Calls Active Simultaneously & 4.17 & 4.17 & 4.31 \\
\hline
\end{tabular}

The network maintains fairness for these calls. This is because Call 1 and Call 2 have the same MOS. Call 3 quality is slightly higher, as expected, due to there only being one call active on that segment of the network. The Min-Max fairness index gives 0.97 as a result. The network is not fair in the treatment of all calls together, but on calls that share the same segments, it is fair.

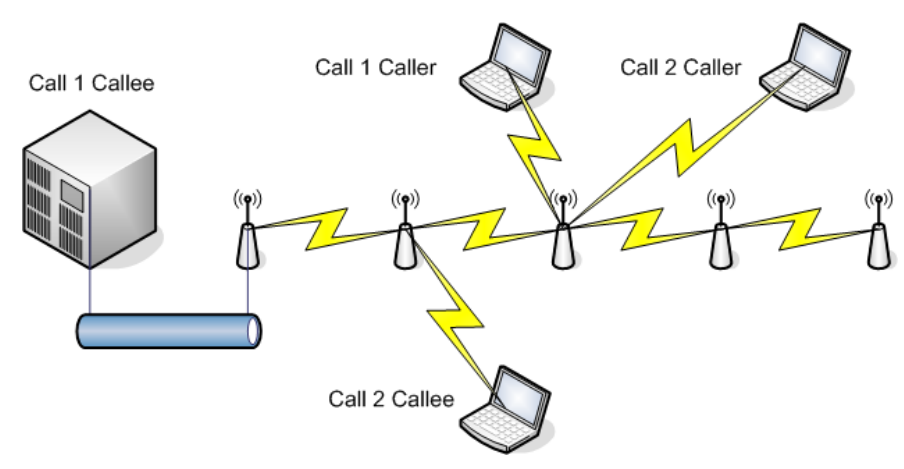

Figure 4.15: Diagram showing the configuration of the network for a fairness test with two calls, where the calls overlap for one hop. 
The fourth fairness test, as shown in Figure 4.15 on the previous page, has two active calls which overlap for one hop on the mesh network. The purpose of this test is to show how the slight overlapping of traffic affects call quality. What is expected here is that the calls show similar performance. Table 4.7 shows the MOS of each call separately and simultaneously.

Table 4.7: Results of Fairness Test of Calls with One Hop Overlap

\begin{tabular}{|c|c|c|}
\hline & MOS of Call 1 & MOS of Call 2 \\
\hline Call 1 Alone & 4.41 & - \\
\hline Call 2 Alone & - & 4.41 \\
\hline Both Calls Active Simultaneously & 4.29 & 4.29 \\
\hline
\end{tabular}

When both calls are active simultaneously, call quality decreases slightly further than when compared to the case with the call paths being adjacent, but not overlapping. This is because the traffic of both calls ends up sharing a wireless link between two of the mesh nodes. The Min-Max fairness index gives 1.00 as a result, showing that the network treats the two calls fairly.

When the MOS numbers of the calls that overlap exactly are compared to those of calls that do not overlap exactly, but do interfere with one another, a call quality decrease is seen. This can be attributed to the fact that increased medium contention exists in the call paths, which is not the case when the call paths overlap exactly.

The fifth fairness test examines the data recorded in the maximum supported calls test to see how fair simultaneous identical calls are. Calls overlap completely and in each case are exactly the same number of hops and traverse the same mesh nodes. What we expect to see in this test is that the calls are not perfectly fair, but 
do not have hugely different MOS values. Table 4.8 below shows individual MOS numbers for each active call, as well as the average for each hop count. For the single hop case, eight calls were active with an average MOS of 3.4 and Min-Max fairness index of 0.95 . The two hop case has an average MOS of 3.7 with a Min-Max fairness index of 0.93. For both the three hop and four hop cases, the average MOS is 4.27 and the Min-Max fairness index is 0.98 and 0.99 respectively. The five hop case, given that there was only one call active, does not have a Min-Max fairness index. The individual MOS numbers for each call are shown in the same column, above the average MOS.

Table 4.8: Results of Fairness of Maximum Supported Calls Test

\begin{tabular}{|c|c|c|c|c|c|}
\hline Call & 1 Hop 1 & 2 Hops & 3 Hops & 4 Hops & 5 Hops \\
\hline 1 & 3.33 & 3.55 & 4.31 & 4.29 & 4.41 \\
\hline 2 & 3.37 & 3.72 & 4.24 & 4.25 & - \\
\hline 3 & 3.51 & 3.80 & 4.28 & - & - \\
\hline 4 & 3.40 & 3.66 & 4.24 & - & - \\
\hline 5 & 3.43 & 3.72 & - & - & - \\
\hline 6 & 3.38 & 3.75 & - & - & - \\
\hline 7 & 3.40 & - & - & - & - \\
\hline 8 & 3.39 & - & - & - & - \\
\hline Average & 3.4 & 3.7 & 4.27 & 4.27 & 4.41 \\
\hline Fairness & 0.95 & 0.93 & 0.98 & 0.99 & - \\
\hline
\end{tabular}

The results show that while the individual call MOS numbers do vary slightly from call to call, no one call stands out as having noticeably better quality or noticeably worse quality. The network treats the calls fairly, as can be seen in the bottom row of the table. Since the QoS parameters are equal for all VoIP traffic, call quality varies only marginally from call to call even with many active simultaneous calls. 


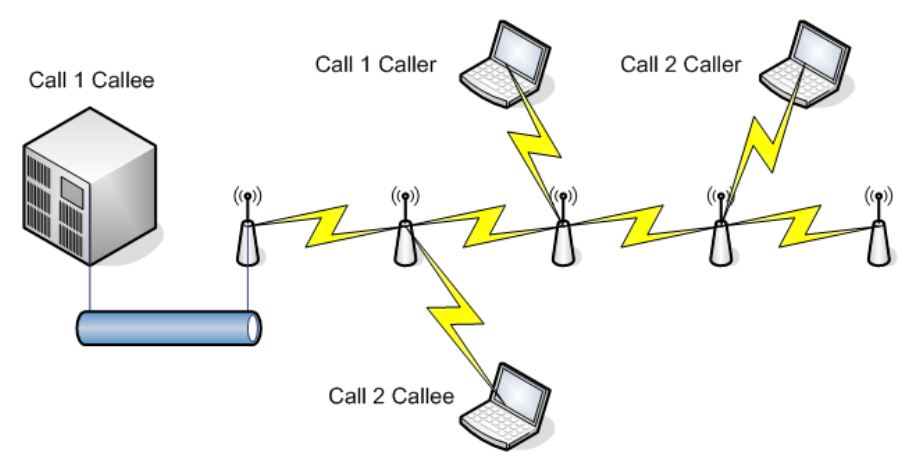

Figure 4.16: Diagram showing the configuration of the network for a fairness test with two calls, where the calls overlap for one hop and are of different hop counts.

The sixth fairness test, as shown in Figure 4.16, tests how fairly the network treats two calls that overlap but are not the same hop count. Call 1 is of hop count three, and Call 2 is of hop count four. As a result, the MOS of Call 2 will be lower than the MOS of Call 1 when both calls are active at the same time. Table 4.9 shows the MOS of each call separately and simultaneously.

Table 4.9: Results of Fairness Test of Calls with Calls of Different Hop Counts

\begin{tabular}{|c|c|c|}
\hline & MOS of Call 1 & MOS of Call 2 \\
\hline Call 1 Alone & 4.41 & - \\
\hline Call 2 Alone & - & 4.41 \\
\hline Both Calls Active Simultaneously & 4.27 & 4.20 \\
\hline
\end{tabular}

The results show a drop in the MOS for the call that traverses more hops, which is in line with our expectations because of increased interference and the additional hop. The Min-Max fairness index gives 0.98 as a result. It is not expected that the calls will show the exact same quality because of the additional hop on one of the calls. 
In summary, the results show that fairness is maintained well across the network with varying call configurations. The network treated calls fairly when expected, showing that the 802.11e QoS mechanism does work.

We now go on to testing how calls in one part of the network affect available bandwidth in other parts of the network.

\subsubsection{Available Bandwidth When Calls Are Active}

We now test how much bandwidth decrease calls cause depending on how far away from other types of traffic they are. The objective of this test is to examine how much of an effect voice calls have on other traffic when the voice calls and other traffic are spaced out, nearby, and adjacent.

The next three tests all have a voice call that must traverse three wireless hops. The fourth test extends the voice call to four hops.

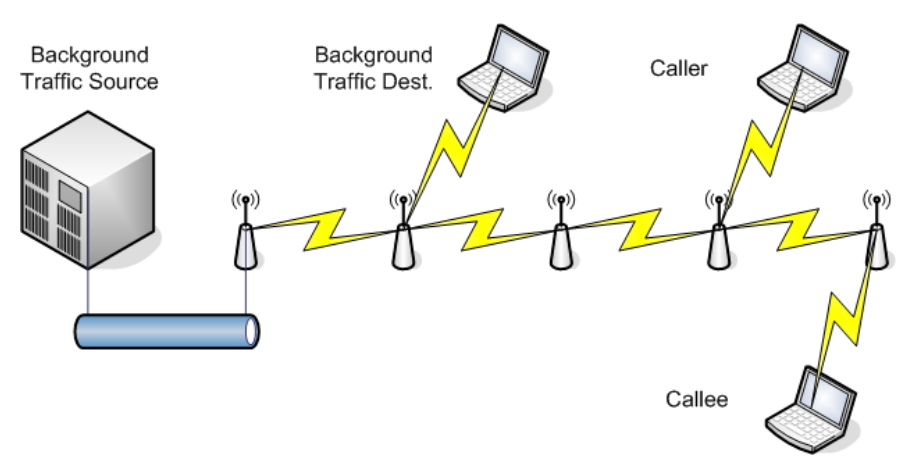

Figure 4.17: Diagram showing background traffic and a single voice call, separated by two hops.

The first of these tests examines the case where background traffic and a voice call are separated by two hops on the mesh network. With this separation, there should be no interference and the performance should be equivalent to a case in 
which the background traffic and voice call are running independently of one another. Figure 4.17 on the previous page shows a diagram of the configuration for this test.

Table 4.10: Results of Available Bandwidth Test with Two Hops Separation

\begin{tabular}{|c|c|c|}
\hline & BG Traffic Throughput & MOS of Call \\
\hline BG Traffic Alone & $3072 \mathrm{kbps}$ & - \\
\hline Call Alone & - & 4.41 \\
\hline Both Active Simultaneously & $3081 \mathrm{kbps}$ & 4.41 \\
\hline
\end{tabular}

The results show that in this case, the background traffic and the voice call do not affect one another. Call quality is equivalent with and without the background traffic running simultaneously. Background traffic throughput is equivalent in both cases as well.

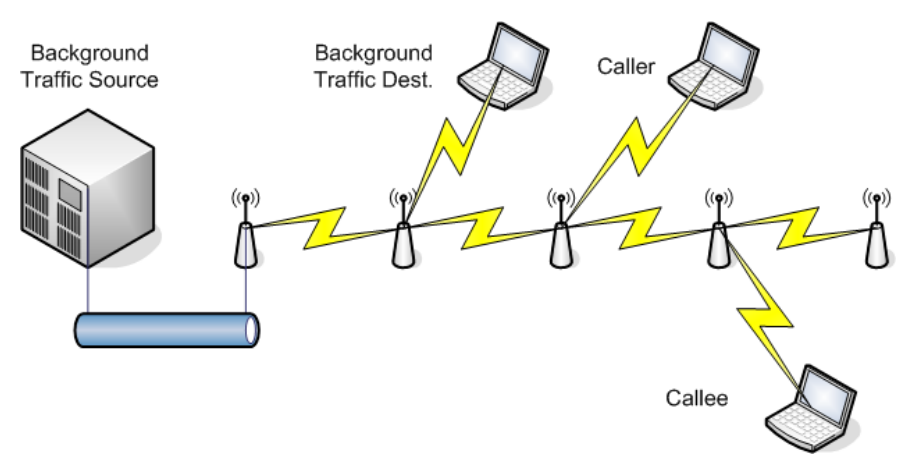

Figure 4.18: Diagram showing background traffic and a single voice call, separated by a single hop.

The second test examines the case where background traffic and a voice call are separated by a single hop on the mesh network. With only a single hop of separation, there is some interference that has an impact on the throughput of the background traffic. Voice call quality should remain fairly constant but is expected to drop off 
slightly due to the added interference. Figure 4.18 on the previous page shows a diagram of the configuration for this test.

Table 4.11: Results of Available Bandwidth Test with One Hop Separation

\begin{tabular}{|c|c|c|}
\hline & BG Traffic Throughput & MOS of Call \\
\hline BG Traffic Alone & $3070 \mathrm{kbps}$ & - \\
\hline Call Alone & - & 4.41 \\
\hline Both Active Simultaneously & $2885 \mathrm{kbps}$ & 4.24 \\
\hline
\end{tabular}

The results show that when simultaneously active, the background traffic and voice call interfere with one another, and this decreases performance. Call quality is still well above the minimum of 3.1. Background traffic throughput only drops off slightly.

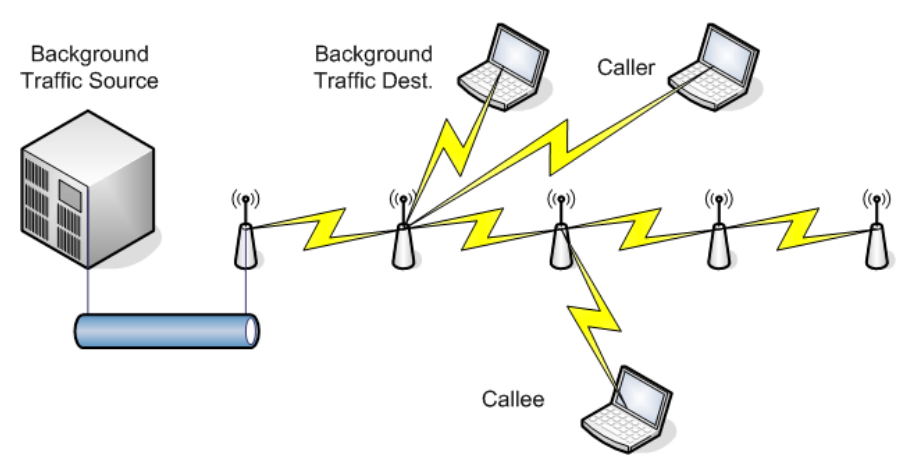

Figure 4.19: Diagram showing background traffic and a single voice call, separated by no space (adjacent).

The third test examines the case where background traffic and a voice call are not separated by any space, that is, they are adjacent to one another. With no separation, interference is greater and performance should be impacted more than in the previous case. Voice call quality is still expected to be very good. Figure 4.19 above shows a 
diagram of the configuration for this test, with no space in between the background traffic and the voice call.

Table 4.12: Results of Available Bandwidth Test with No Separation (Adjacent)

\begin{tabular}{|c|c|c|}
\hline & BG Traffic Throughput & MOS of Call \\
\hline BG Traffic Alone & $3079 \mathrm{kbps}$ & - \\
\hline Call Alone & - & 4.41 \\
\hline Both Active Simultaneously & $2507 \mathrm{kbps}$ & 4.14 \\
\hline
\end{tabular}

The results show that when both the background traffic and voice call are simultaneously active, the interference impacts performance. Call quality is still well above the 3.1 minimum at 4.14 and background traffic throughput has only been impacted slightly, from $3079 \mathrm{kbps}$ to $2507 \mathrm{kbps}$.

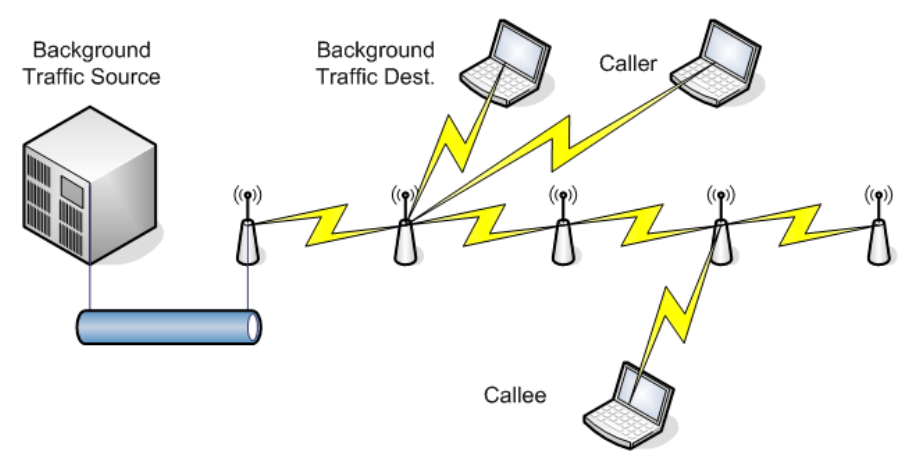

Figure 4.20: Diagram showing background traffic and a single voice call, separated by no space (adjacent).

The fourth test examines the same case as the previous test, but the call length is four hops instead of three. The purpose of this test is to show that the background traffic does not impact call quality when the call is further extended beyond the 
interference reach of the background traffic. Figure 4.20 on the previous page shows a diagram of the configuration for this test.

Table 4.13: Results of Available Bandwidth Test with No Separation (Adjacent) but Four Hops

\begin{tabular}{|c|c|c|}
\hline & BG Traffic Throughput & MOS of Call \\
\hline BG Traffic Alone & $3077 \mathrm{kbps}$ & - \\
\hline Call Alone & - & 4.41 \\
\hline Both Active Simultaneously & $2514 \mathrm{kbps}$ & 4.13 \\
\hline
\end{tabular}

The results show that increasing the number of hops of the voice call does not have an effect on the background traffic as long as the background traffic is not within hearing range of the additional hop. The call has been extended beyond the range that the background traffic would be effected by, so the additional hop does not interfere at all with the background traffic. The throughput of the background traffic is not effected, at $2514 \mathrm{kbps}$ instead of $2507 \mathrm{kbps}$.

In summary, we studied the effect of calls on available bandwidth on the network. We did this by separating calls and background traffic first by two hops, then by one hop, then by having them adjacent to one another. Following this, we extended the call length by one hop to test if the additional hop of the call outside the interference range of the background traffic would effect the background traffic at all.

In the first part of this test, with a call spaced from the background traffic by two hops, the MOS of the call was 4.41, and the background traffic 3081 kbps. When the space was decreased to one hop, the MOS of the call was 4.24 and the background traffic $2885 \mathrm{kbps}$. This is a drop of $4 \%$ in the call quality and $6 \%$ in the speed of the background traffic. When the space between the call and the background traffic was 
decreased to zero hops, that is, adjacent to one another, the MOS of the call dropped to 4.14 and the background traffic 2507 . This represents a further drop of $2 \%$ for the call quality and $13 \%$ for the background traffic. When stacked, this represents a total drop of $6 \%$ for the call quality and $19 \%$ for the background traffic when going from no interference to adjacent and interfering.

The final part of this test extended the length of the call by one hop. The extra hop on the call did not affect the background traffic throughput at all because the extra hop was outside of the hearing range of the background traffic.

\subsubsection{Simultaneous Calls with Different QoS Parameters}

This section examines call quality when some calls have QoS enabled while others do not. The calls are placed in different QoS queues by changing the DSCP flag in both X-Lite, the software SIP phone, and the Asterisk server. The non-QoS enabled calls are placed in the background traffic queue, while the QoS enabled calls are placed in the voice queue.

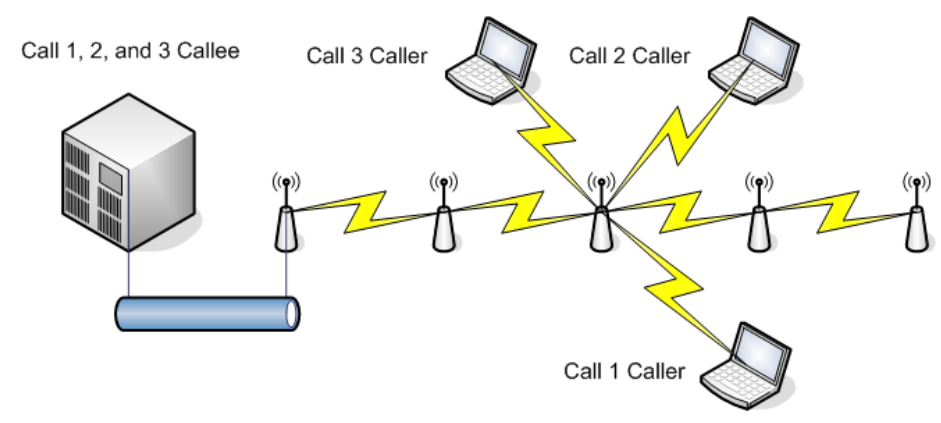

Figure 4.21: Diagram showing three overlapping calls.

In the first test of this section, we examine call quality of overlapping calls. Some have QoS enabled, while others do not. With all calls simultaneously active, we 
expect to see the QoS enabled calls having a higher MOS value than the non-QoS enabled calls. When only one of the calls is active, we expect to see perfect performance regardless of the QoS setting. A diagram of this test configuration is shown in Figure 4.21 on the previous page. The details of which are QoS-enabled are contained in Table 4.14 below.

Table 4.14: Results of Call Test, Overlapping Calls, Different QoS Settings

\begin{tabular}{|l|c|c|c|}
\hline & MOS of Call 1 & MOS of Call 2 & MOS of Call 3 \\
\hline Call 1 Alone & 4.41 & - & - \\
\hline Call 2 Alone & - & 4.41 & - \\
\hline Call 3 Alone & - & - & 4.41 \\
\hline All Calls Simul. with QoS & 4.34 & 4.34 & 4.33 \\
\hline All Calls Simul. with 1 QoS & 4.40 & 4.12 & 4.13 \\
\hline $\begin{array}{l}\text { All Calls Simul. with 1 QoS, and } \\
\text { Background }\end{array}$ & 4.37 & 3.80 & 3.76 \\
\hline All Calls Simul. with 1, 2 QoS & 4.37 & 4.36 & 4.09 \\
\hline $\begin{array}{l}\text { All Calls Simul. with 1, 2 QoS, } \\
\text { and Background }\end{array}$ & 4.30 & 4.30 & 3.42 \\
\hline
\end{tabular}

The results of this test confirm that some traffic types may be treated differently depending on what QoS queue they are placed in. When all the calls have QoS enabled, the MOS values are approximately equal, as they should be. When only the first call has QoS enabled, and the other two calls do not, the first call MOS value is quite a lot higher than the other two calls. This shows that the QoS is working and the packets of the first call are being prioritized over the packets of the other two calls. When the first two calls have QoS and the third call does not, the third call has a slightly lower MOS than the case when only one call has QoS enabled. This happens because two active calls with QoS use more of the available bandwidth and 
leave less for the third call.

When background traffic is also added, the quality of the calls that are not QoS enabled drops significantly, because the voice traffic and background traffic are being put together in the same queue. This is evident in both cases tested, when only the first call is QoS enabled, and when both the first and second calls are QoS enabled.

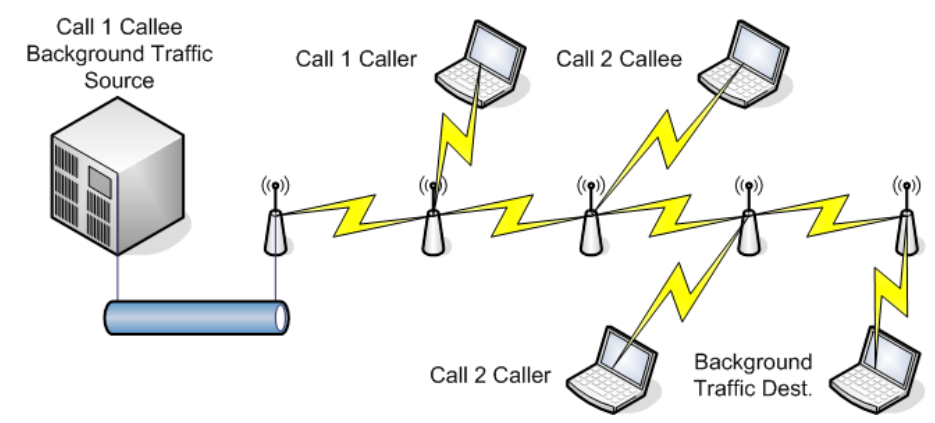

Figure 4.22: Diagram showing two calls running on separate parts of the network.

In the second test of this section, we examine call quality for two calls that are separated by one hop on the network. Each call is a three hop call. Background traffic between both ends of the network is added for some of the test cases. Table 4.15 on the next page below contains the results of this test and details which calls have QoS, and if background traffic is present. A diagram of the network configuration for this test is in Figure 4.22 above. 
Table 4.15: Results of Call Test, Separate Calls, Different QoS Settings

\begin{tabular}{|l|c|c|}
\hline & MOS of Call 1 & MOS of Call 2 \\
\hline Call 1 Alone & 4.41 & - \\
\hline Call 1 Alone with Background & 4.27 & - \\
\hline Call 2 Alone & - & 4.41 \\
\hline Call 2 Alone with Background & - & 3.96 \\
\hline All Calls Simul. with QoS on Both & 4.41 & 4.41 \\
\hline All Calls Simul. with 1 QoS & 4.41 & 4.41 \\
\hline All Calls Simul. with 1 QoS, and Background & 4.27 & 3.95 \\
\hline
\end{tabular}

As is seen in the table of results, when the calls are active simultaneously, the call quality is the same. This is because the calls are not traversing the same part of the network. As a result, they are not competing for access to the medium from two different QoS traffic queues. However, when adding background traffic traversing the entire length of the network, the voice traffic of the second call competes with the background traffic as it is in the same queue. As a result, the MOS value of the second call drops off significantly more when background traffic is added than the MOS value of the first call does. This shows that the QoS mechanism is working correctly and as expected in this situation.

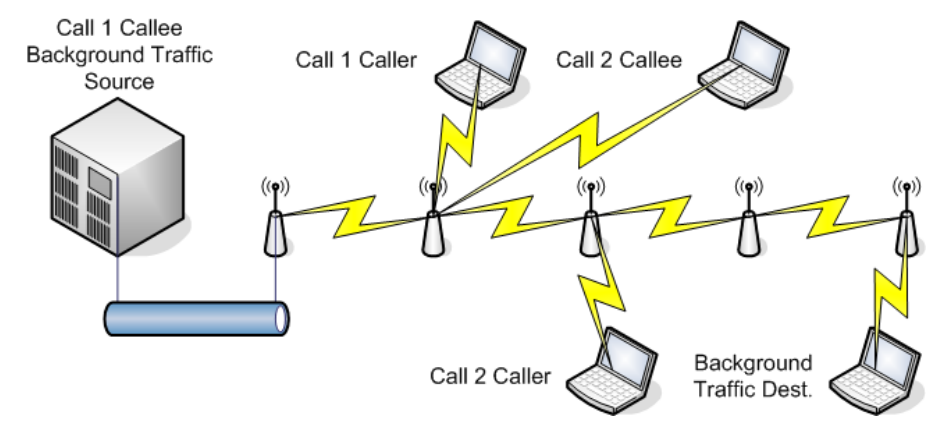

Figure 4.23: Diagram showing two calls that are adjacent to one another. 
In the third test of this section, we examine call quality for two calls that are adjacent to one another. Background traffic between both ends of the network is added for some of the test cases. Table 4.16 contains the results of this test and details which calls have QoS, and if background traffic is present. The network configuration for this test is shown in Figure 4.23 on the previous page.

Table 4.16: Results of Call Test, Adjacent Calls, Different QoS Settings

\begin{tabular}{|l|c|c|}
\hline & MOS of Call 1 & MOS of Call 2 \\
\hline Call 1 Alone & 4.41 & - \\
\hline Call 1 Alone with BG & 4.27 & - \\
\hline Call 2 Alone & - & 4.41 \\
\hline Call 2 Alone with BG & - & 3.95 \\
\hline All Calls Simul. with QoS on Both & 4.41 & 4.41 \\
\hline All Calls Simul. with 1 QoS & 4.41 & 4.36 \\
\hline All Calls Simul. with 1 QoS, and BG & 4.27 & 3.88 \\
\hline
\end{tabular}

As shown in the table of results, because one node is handling the traffic of both calls, call quality of the non-QoS enabled call decreases slightly further. This occurs whether there is background traffic or not. As the calls get closer together, like in this case, the QoS enabled call has more of an impact on the non-QoS enabled call. This shows that the QoS-enabled call is being favoured as expected. The drop in call quality is expected when compared to the previous test, where there was a single hop space between the two calls.

In summary, we examined how calls perform when different calls have different QoS settings. With three calls that overlap exactly, the call quality varied significantly depending on which calls had QoS enabled and which did not. For example, when Call 1 was QoS-enabled while Call 2 and Call 3 were not, they experienced a call 
quality $6 \%$ lower than Call 1. For full details, revisit Table 4.14 on page 68.

With the second part of this test, we tested call quality of two calls with spacing between them. When both calls are active simultaneously, they do not effect one another, and as a result, the MOS is high for both calls at 4.41 in the case where one call is QoS-enabled and the other is not. When background traffic is added, both call qualities decrease. The call that is not QoS-enabled has a call quality MOS that is 7\% lower than the QoS-enabled call.

With the third part of this test, we tested call quality of two adjacent calls, one that was QoS-enabled, and one that was not. When both calls are active and there is no background traffic, call quality MOS of the not QoS-enabled call is $1 \%$ lower than the QoS-enabled call. With background traffic added, this number increases to $9 \%$.

\subsubsection{Effect of Bottleneck Node}

The next test loads a single node with traffic to examine how it effects call quality and call performance.

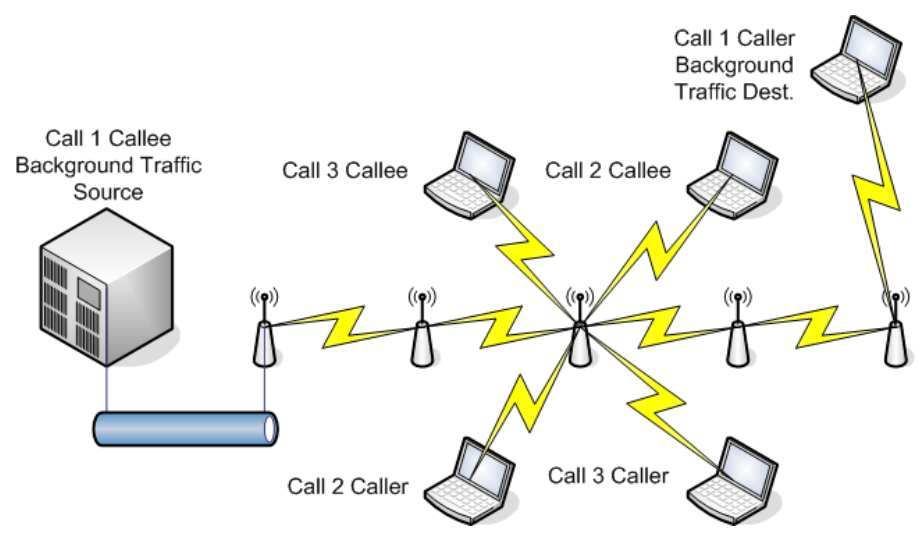

Figure 4.24: Diagram showing three calls that all pass through a central node. 
This test examines call quality when a central node is handling a large amount of traffic. In this case, two calls with two hop count are configured to pass through the node, as is a third call with five hop count. Background traffic is also added. Details about the active calls and background traffic are shown along with the results, in Table 4.17. A diagram of the configuration for this test can be found in Figure 4.24 on the previous page.

Table 4.17: Results of Call Test, Bottleneck Node

\begin{tabular}{|l|c|c|c|}
\hline & MOS of Call 1 & MOS of Call 2 & MOS of Call 3 \\
\hline Call 1 Alone & 4.41 & - & - \\
\hline Call 1 with BG Traffic & 3.90 & - & - \\
\hline Call 2 Alone & - & 4.41 & - \\
\hline Call 3 Alone & - & - & 4.41 \\
\hline All Calls Simultaneously & 4.19 & 4.38 & 4.38 \\
\hline $\begin{array}{l}\text { All Calls Simultaneously with BG } \\
\text { Traffic }\end{array}$ & 3.64 & 4.24 & 4.23 \\
\hline
\end{tabular}

As can be seen, when the first call is active with background traffic, the MOS value is 3.90. When the additional calls are added at the central node, the quality of the first call is decreased even further because of the extra traffic and medium contention at the central node where the second and third call take place. The additional traffic at the central node does impact performance, as is expected. The same degradation in quality of the first call takes place when there is no background traffic as well, but it does not have as large an effect without the background traffic due to the decreased medium contention and lower utilization on the network.

In summary, we examined how call quality is when a single central node is handling 
much of the traffic. What we found is that the long hop count call is affected the most when the centralized calls are active, because of the extra medium contention in that region of the network.

\subsection{Summary}

Recall the n-Hops test, where we tested voice call quality with varying numbers of hops from one to five on the WMN. We looked at one call and two calls, both with and without background traffic, while adjusting the CWmax parameter for the voice traffic and the AIFS parameter for the background traffic. In our testing, a CWmax of $63 \mathrm{~ms}$ and AIFS of $15 \mathrm{~ms}$ gave us the highest call quality of all the parameter value combinations we tried.

In the maximum calls test, we tested how many calls can be supported simultaneously at different hop counts. Our results are summarized in Table 4.18 below.

Table 4.18: Maximum Number of Supported Calls

\begin{tabular}{|c|c|c|c|c|c|}
\hline Hop Count & 1 Hop & 2 Hops & 3 Hops & 4 Hops & 5 Hops \\
\hline Maximum Number of Calls & 8 & 6 & 4 & 2 & 1 \\
\hline
\end{tabular}

In the fairness test, we studied how well the network maintains fairness between different calls. Calls were maintained fair by the network when expected, showing that the 802.11e QoS mechanism does treat calls fairly when able to.

In the available bandwidth test, we studied the effect of varying numbers of calls on available bandwidth in adjacent portions of the network. We first separated calls by two hops, then one hop, and then having them adjacent to one another. Results 
showed that background traffic throughput dropped by a total of $19 \%$ when the call was moved from two hops distance from the background traffic to adjacent to the background traffic.

In the different QoS settings test, we looked at call quality when different calls have different QoS settings. Calls placed in the voice traffic class had higher call quality than calls that were placed in the background traffic class.

In the bottleneck test, we examined how calls perform when a single central node is handing a large percentage of the traffic on the network. We found that calls that pass through the busy central node are affected by the additional traffic passing through this node.

The experiments reveal that the 802.11e QoS mechanism can support the desired QoS for voice calls. When compared with call quality when 802.11e QoS is disabled, the call quality is improved on average, in all cases except for the one where the CWmax of the voice traffic class is set to $7 \mathrm{~ms}$. In this case, the low CWmax setting has a large effect on decreasing the call quality for the four and five hop tests. In addition, the experiments revealed that the default EDCA parameters for the 802.11e QoS mechanism are not suitable on a multi-hop mesh network. Modified parameter settings have been provided and used in the testing. With these settings, increased call quality is observed over multiple hops. It is important to note, however, that voice over an 802.11 mesh network is not really practical to deploy except in a very small user base because the number of calls that can be supported is limited.

When the new parameter settings are used in the single hop case, the voice call quality is not adversely affected by a large amount, but, the CWmax setting for voice should be kept at the minimum possible value for any given hop count. The CWmax 
and AIFS setting we used were chosen by testing all different combinations of both parameters. 


\section{Chapter 5}

\section{Conclusion}

In recent years, VoIP has been increasing in popularity. Convergence of communications onto IP networks is happening everywhere, with video, voice, and other realtime services being sent over the same networks as other data, such as web and E-mail traffic. Different services have different delay tolerances and requirements to work properly. The IEEE 802.11 e amendment to the IEEE 802.11 standard adds support for QoS to better support multiple services concurrently.

In this thesis, we focused on the ability of 802.11 e to meet the QoS requirements for voice calls on a WMN. We gave background on the 802.11 protocol, WMNs, VoIP, SIP, and discussed some other VoIP performance works. We discussed our implementation, which includes an experimental WMN testbed used for testing call quality. Following this, we discussed the way we record and process the data. We then evaluated call quality in a variety of configurations and different scenarios on our WMN testbed. We identified performance metrics to use for studying the quality of VoIP calls. We detailed the individual test scenarios and presented the results and conducted a series of tests to examine if the 802.11e QoS mechanism provides any 
improvement over the original 802.11 standard.

Voice quality was measured using recorded data about end-to-end delay, jitter, and packet loss. A comparable measure was calculated by using the E-model.

The 802.11e QoS mechanism does help improve call quality, however the default settings cause problems with mesh networks. To improve voice call quality with a similar configuration as our wireless mesh network testbed, we recommend the following:

- Configuring the AIFS parameter for the background and best effort traffic classes to a higher than default value. Doing this desensitizes voice traffic from background traffic without impacting background traffic throughput. In our research, we used the value of $15 \mathrm{~ms}$. If it was set higher, background traffic throughput was noticeably impacted.

- Configuring the CWmax parameter for the voice traffic class to a higher value than the default one. Doing this decreases the contention on the wireless medium over multiple hops and helps increase the voice call quality at longer hop counts without causing excessive delays. In our research, we used the value of $63 \mathrm{~ms}$. When the CWmax value was set higher, further improvement in call quality or background traffic throughput was not seen.

- Configuring a fixed transmission rate of at least $11 \mathrm{Mbps}$ so that MAC-level errors do not automatically drop the rate too low.

- Ensuring $802.11 \mathrm{e}$ is enabled between mesh routers by using a wireless sniffer. Configuring mesh routers with DD-WRT in Client Bridge mode accomplishes this. 
Using these guidelines will help to improve call quality on WMNs. Fairness will be maintained on the network when comparing similar types of calls. In addition, background traffic throughput will not be severely impacted.

In reality, VoIP over 802.11 mesh networks is not very practical, especially in extended hop cases. Actual deployment to serve a large number of users will require provisions to accomdate a higher number of calls that can be supported simultaneously. In order to improve the voice call quality more, further study is needed. In particular, future work can be directed at the following areas:

- Voice call quality over mesh networks with different backhaul network types should be studied. This includes dual-radio routers that have either two $802.11 \mathrm{~b} / \mathrm{g}$ radios or one $802.11 \mathrm{a}$ and one $802.11 \mathrm{~b} / \mathrm{g}$ radio. A multi-radio $802.11 \mathrm{~b} / \mathrm{g}$ router could improve performance as two separate channels would result in less interference and less overall contention for the wireless medium. A mixed 802.11a and $802.11 \mathrm{~b} / \mathrm{g}$ router could improve performance by having the access portion of the network run on $802.11 \mathrm{~b} / \mathrm{g}$ while running the backhaul portion on 802.11a. These routers are not as widely available and the cost per router is much higher.

- A call admission control system could be implemented to ensure that no more calls than can be supported are permitted on the network at any time. This type of system would monitor network conditions and only permit as many calls at any given time as can be supported. As was seen in the test of the maximum number of supported calls, one additional call that goes beyond what the network can support drops the call quality of all calls significantly.

The devices and software we used in this study have some limitations. They are as follows: 
- Our use of DD-WRT does not allow us to see all network information transparently. We are not able to see per-node statistics on traffic classes. Instead, we have to gather this information by studying the network traffic.

- The use of single radio routers limits our ability to test further, as mentioned above. 


\section{Bibliography}

[1] I. Akyildiz and X. Wang. A Survey on Wireless Mesh Networks. IEEE Radio Communications, 43(9):S23-S30, 2005.

[2] M. Alexander and P. Suppan. An Architecture for SDP-Based BAndwidth Resource Allocation with QoS for SIP. ACM International Workshop on Quality of Service and Security for Wireless and Mobile Networks, pages 75-82, October 2006.

[3] F. Anjum, M. Elaoud, D. Famolari, A. Ghosh, R. Vaidyanathan, A. Dutta, and P. Agrawal. Voice Performance in WLAN Networks - An Experimental Study. Globecom, pages 3504-3508, 2003.

[4] L. Atzori and M. Lobina. Playout Buffering in IP Telephony: A Survey Discussing Problems and Approaches. IEEE Communications Surveys and Tutorials, Third Quarter 2006.

[5] S. Blake, D. Black, M. Carlson, E. Davies, Z. Wang, and W. Weiss. An Architecture for Differentiated Service. RFC 2475, Internet Engineering Task Force, December 1998. 
[6] S. Cherry. Seven Myths About Voice over IP. IEEE Spectrum, 42(3):52-57, March 2005.

[7] G. Combs. Wireshark - Network Protocol Analyzer. http://www.wireshark.org/.

[8] CounterPath. X-Lite - Software SIP Phone. http://www.counterpath.com/xlite.html.

[9] S. Das, E. Lee, K. Basu, and S. Sen. Performance Optimization of VoIP Calls over Wireless Links Using H.323 Protocol. IEEE Transactions on Computers, 52(6):742-752, June 2003.

[10] M. Dianati, X. Shen, and S. Naik. A New Fairness Index for Radio Resource Allocation in Wireless Networks. IEEE Wireless Communications and Networking Conference, pages 712-717, March 2005.

[11] Digium. Asterisk - The Open Source PBX and Telephony Platform. http://www.asterisk.org/.

[12] S. Ganguly, V. Navda, K. Kim, A. Kashyap, D. Niculescu, R. Izmailov, S. Hong, and S. Das. Performance Optimizations for Deploying VoIP Services in Mesh Networks. IEEE Journal on Selectted Areas in Communications, 24(11):21472157, November 2006.

[13] S. Garg and M. Kappes. An Experimental Study of Throughput for UDP and VoIP Traffic in IEEE 802.11b Networks. Wireless Communications and Networking, 3:1748-1753, March 2003.

[14] J. Gibson. Speech Coding Methods, Standards, and Applications. IEEE Circuits and Systems Magazine, 5(4):30-49, Fourth Quarter 2005. 
[15] B. Goode. Voice over Internet Protocol. Proceedings of the IEEE, 90(9):14951517, September 2002.

[16] S. Gottschall, C. Scheele, A. Majdic, S. Bothorel, and F. Fietkau. DD-WRT. http://www.dd-wrt.com/.

[17] M. Handley, H. Schulzrinne, E. Schooler, and J. Rosenberg. SIP: Session Initiation Protocol. RFC 2543, Internet Engineering Task Force, March 1999.

[18] G. Hanley, S. Murphy, and L. Murphy. Adapting WLAN MAC Paramters to Enhance VoIP Call Capacity. International Symposium on Modeling, Analysis and Simulation of Wireless and Mobile Systems, pages 250-254, October 2005.

[19] Digium Inc. http://www.digium.com/en/index.php.

[20] K. Kim and S. Hong. VoMESH: Voice over Wireless Mesh Networks. Proceedings of Wireless Communications and Networking Conference, 1:193-198, April.

[21] Linksys. http://www.linksys.com/.

[22] LinuxOnline. Linux Operating System. http://www.linux.org/.

[23] M. Maresca, N. Zingirian, and P. Baglietto. Internet Protocol Support for Telephony. Proceeding of the IEEE, 92(9):1463-1477, September 2004.

[24] N. Nandiraju, D. Nandiraju, L. Santhanam, B. He, J. Wang, and D. Agrawal. Wireless Mesh Networks: Current Challenges and Future Directions of Web-inthe-Sky. IEEE Wireless Communications, 14(4):79-89, August 2007. 
[25] M. Narbutt and M. Davis. Gauging VoIP Call Quality from 802.11 WLAN Resource Usage. International Symposium on a World of Wireless, Mobile and Multimedia Networks, pages 315-324, 2006.

[26] M. Narbutt and M. Davis. Experimental Tuning of AIFSN and CWmin Parameters to Prioritize Voice of Data Transmission in 802.11e WLAN Networks. International Wireless Communications and Mobile Computing Conference, pages 140-145, August 2007.

[27] K. Nichols, S. Blake, F. Baker, and D. Black. Definition of the Differentiated Services Field (DS Field) in the IPv4 and IPv6 Headers. RFC 2474, Internet Engineering Task Force, December 1998.

[28] D. Niculescu, S. Ganguly, Kyungtae, and Rauf Izmailov. Performance of VoIP in a 802.11 Wireless Mesh Network. IEEE Infocom, pages 1-11, November 2006.

[29] Y. Qian, R. Hu, and H. Chen. A Call Admission Control Framework for Voice over WLANs. IEEE Wireless Communications, 13(1):44-50, February 2006.

[30] J. Rosenberg, H. Schulzrinne, G. Camarillo, A. Johnston, J. Peterson, R. Sparks, M. Handley, and E. Schooler. SIP: Session Initiation Protocol. RFC 3261, Internet Engineering Task Force, June 2002.

[31] H. Schulzrinne, S. Casner, R. Frederick, and V. Jacobson. RTP: A Transport Protocol for Real-Time Applications. RFC 3550, Internet Engineering Task Force, July 2003.

[32] IEEE Computer Society. IEEE Std 802.11-2007, revision of IEEE Std 802.111999. Technical report, IEEE Computer Society, 2007. 
[33] UCDavis. WinPcap: The Windows Packet Capture Library. http://www.winpcap.org/.

[34] International Telecommunications Union. Methods for subjective determination of transmission quality. Technical report, International Telecommunications Union, Telecommunication Standardization Sector, August 1996.

[35] International Telecommunications Union. Appendix I: Provisional planning values for the equipment impairment factor Ie, ITU-T Recommendation G.113. Technical report, International Telecommunications Union, Telecommunication Standardization Sector, December 1998.

[36] International Telecommunications Union. Definition of categories of speech transmission quality, ITU-T Recommendation G.109. Technical report, International Telecommunications Union, Telecommunication Standardization Sector, September 1999.

[37] International Telecommunications Union. The E-model, a computational model for use in transmission planning, ITU-T Recommendation G.107. Technical report, International Telecommunications Union, Telecommunication Standardization Sector, March 2005.

[38] P. Wang, H. Jiang, and W. Zhuang. IEEE 802.11e Enhancement for Voice Services. IEEE Wireless Communications, 13(1):30-35, February 2006.

[39] W. Wang, S. Liew, and V. Li. Solutions to Performance Problems in VoIP Over a 802.11 Wireless LAN. IEEE Transactions on Vehicular Technology, 54(1):366384, January 2005. 


\section{Appendix A}

\section{DD-WRT}

DD-WRT is a free Linux-based firmware for different wireless routers based on Atheros or Broadcom wireless chipsets. Many features provided by DD-WRT have parameters that we adjusted during our testing. Many of DD-WRTs features are not provided in the normal firmware that comes with the routers. These features include modes other than the default access point mode, such as client mode, bridge mode, and client bridge mode. Also, daemon-based services such as a VPN server, SMB server, and others are included. In addition, IPv6 support is available, as is RADIUS, and WDS (wireless distribution system). The use of DD-WRT was a natural choice for

our research, as it allowed us to use standard Linksys routers as mesh routers and clients with QoS support.

The screenshots below show the Advanced Wireless configuration page, allowing for configuration of rate, wireless settings, and QoS settings. 


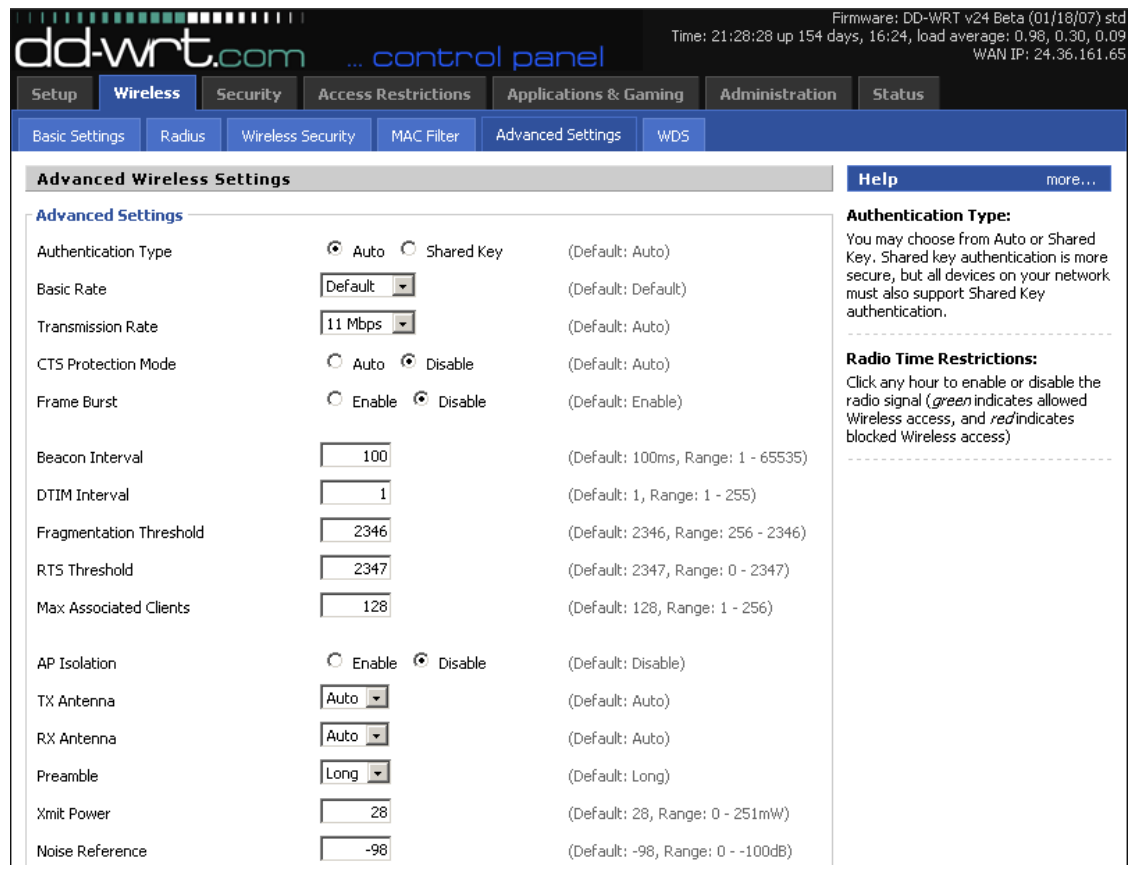

Figure A.1: DD-WRT Wireless Configuration Page

The above screenshot shows the configuration page for setting various wireless options. The DD-WRT software allows one to modify just about any parameter, thereby allowing for many different options for testing. While we did not actually adjust many of the parameters found on this page, this is a good sampling of what can be changed, and how the DD-WRT interface is laid out. 


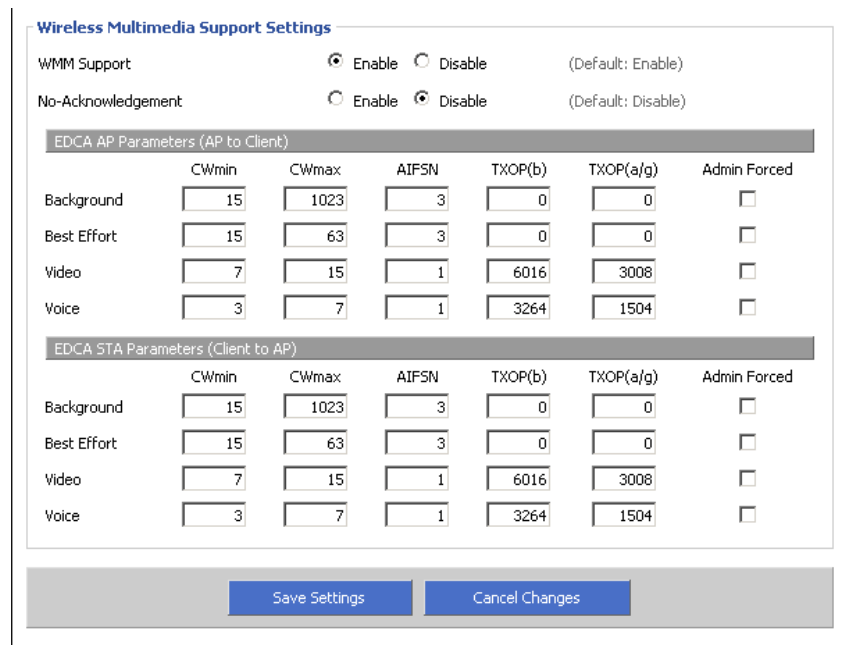

Figure A.2: DD-WRT QoS Configuration

The above screenshot shows the QoS configuration page, where we adjusted numerous parameters to test various QoS settings. These parameters can have a great effect on the call quality and background traffic throughput.

Our choice of DD-WRT as a firmware was based on the fact that it supports QoS, allows for configuration as a mesh router, and allows parameters to be changed as needed. With this, we were able to construct a wireless mesh network testbed and use it to test call quality in real situations. 


\section{Appendix B}

\section{E-model}

The E-model is a transmission planning tool developed by the ITU-T in recommendation G.107 [37]. It provides a prediction of the expected voice quality as would be perceived by a typical telephone user participating in a complete end-to-end voice call. The E-model takes into account a wide range of impairments, such as CODEC impairments, end-to-end delay, jitter, and packet loss, as well as noise and echo. The last two, noise and echo, are not studied in our research because they are not affected by our wireless mesh network.

The ITU-T E-model is based on modeling the results of a large number of subjective tests done to measure perceived voice call quality. It is not a true model in the sense that it cannot accurately predict the absolute opinion of an individual user, but, over a large number of users, the results are sufficiently accurate to permit use for planning and evaluation purposes. The output of the E-model is a value known as the R-value, or Transmission Rating Factor. This can be transformed into other quality measures, such as MOS.

The E-model is based on a mathematical algorithm. The individual transmission 
parameters are transformed into different impairment factors that are combined to produce the R-value ranging from 0 to 100.

$$
R=R o-I s-I d-I e E f f+A
$$

Where Ro expresses the basic signal to noise ratio, Is represents all impairments that occur more or less simultaneously with the voice signal (such as too loud speech, excessive noise), Id represents the sum of all impairments due to delay, echo and jitter effects, IeEff represents the impairment caused by CODEC choice and packet loss, and $\mathbf{A}$ represents the advantage factor, used to offset additional impairment caused by connections such as multi-hop satellite networks.

In our research, we are only concerned with Id and IeEff impairments, which include delay, jitter, packet loss and CODEC choice. These are the only impairments that are affected by our network and our choices.

IeEff, the equipment impairment factor, is calculated as follows. In this formula, Ie is $0, \mathbf{B p l}$ is 0.15 , and the maximum value of $\mathrm{R}$ is 92.21 , as defined in [35] for the G.711 CODEC.

$$
I e E f f=I e+(95-I e) \times \frac{P p l}{\frac{P p l}{B u r s t R}+B p l}
$$

where Burst $R=1$ for random packet loss

and $P p l=$ the packet loss probability rate, in $\%$

Id, the delay impairment factor, is calculated as follows.

$$
I d=I d t e+I d l e+I d d+I j
$$


where Idte gives an estimate for the impairments due to talker echo, Idle represents impairments due to listener echo, and Idd represents the impairment caused by too-long absolute delay Ta.

For $T a \leq 100 m s, I d d=0$

$$
\begin{gathered}
\text { For } T a>100 m s, I d d=25 \times\left(\left(1+X^{6}\right)^{1 / 6}-3\left(1+\frac{X^{6}}{3}\right)^{1 / 6}+2\right) \\
\text { with } X=\frac{\log \frac{T a}{100}}{\log 2}
\end{gathered}
$$

For $\mathbf{I} \mathbf{j}$, jitter, the same calculation is performed, with one exception. Ta is replaced with TaJ, and is calculated as follows.

$$
\text { TaJ }=T a+(2 \times j i t t e r)
$$

Following the calculation of TaJ, the same calculations as above are carried out, replacing Ta with TaJ.

Once an R-value has been calculated, an estimated MOS can be calculated for the voice call quality using the formulae that follow.

$$
\text { For } R<0, M O S=1
$$

$$
\text { For } 0<R<100, M O S=1+0.035 R+R(R-60)(100-R) 7 \times 10^{-6}
$$

For $R>100, M O S=4.5$

This is the calculation we use to calculate MOS values for test calls in our mesh network testbed. The below figure shows MOS as a function of the R-value, from [37], the result of the $0<R<100$ MOS formula. 


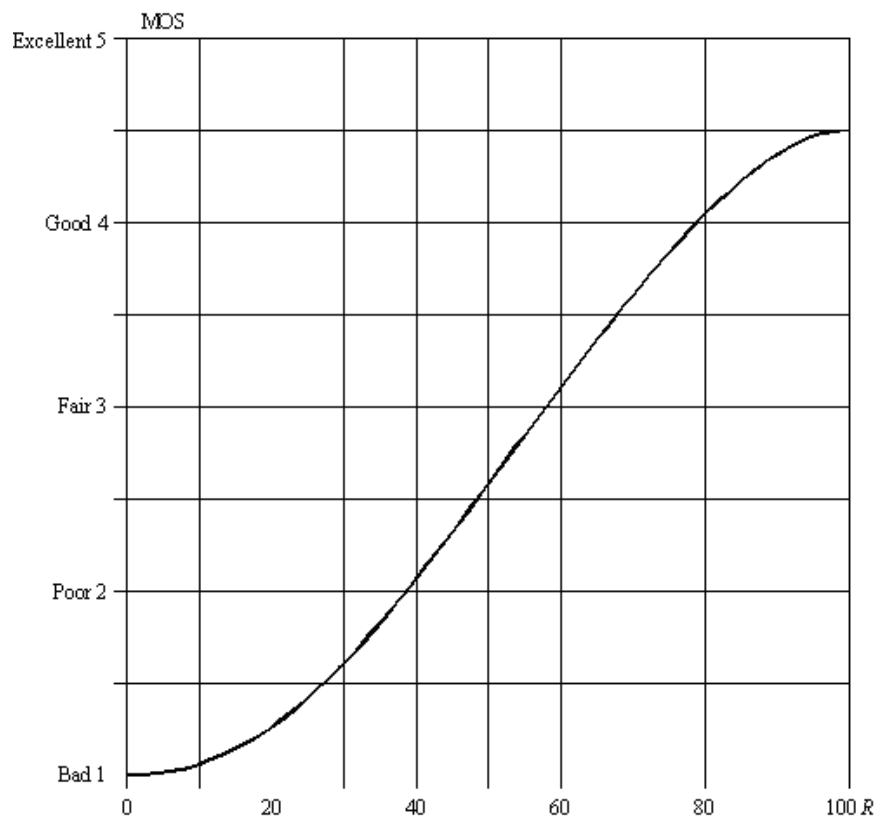

Figure B.1: MOS as a Function of the R-Value 